\title{
1 Machine learning sequence prioritization for cell type-specific enhancer design
}

2

3 Alyssa J Lawler ${ }^{1,2,3}$, Easwaran Ramamurthy ${ }^{1,3}$, Ashley R Brown ${ }^{1,3}$, Naomi Shin ${ }^{1,3}$, Yeonju Kim ${ }^{1,3}$, Noelle

4 Toong ${ }^{1,3}$, Irene M Kaplow ${ }^{1,3}$, Morgan Wirthlin ${ }^{1,3}$, Xiaoyu Zhang ${ }^{1,3}$, Grant Fox ${ }^{1,3}$, Andreas R Pfenning*1,2,3

$5{ }^{1}$ Computational Biology Department, School of Computer Science, Carnegie Mellon University,

6 tPittsburgh, PA, USA

$7 \quad{ }^{2}$ Biological Sciences Department, Mellon College of Science, Carnegie Mellon University, Pittsburgh,

8 PA, USA

$9 \quad{ }^{3}$ Neuroscience Institute, Carnegie Mellon University, Pittsburgh, PA, USA

$10 *$ Corresponding author

11

12 Abstract

Recent discoveries of extreme cellular diversity in the brain warrant rapid development of

14 technologies to access specific cell populations, enabling characterization of their roles in behavior and in

15 disease states. Available approaches for engineering targeted technologies for new neuron subtypes are

16 low-yield, involving intensive transgenic strain or virus screening. Here, we introduce SNAIL (Specific

17 Nuclear-Anchored Independent Labeling), a new virus-based strategy for cell labeling and nuclear

18 isolation from heterogeneous tissue. SNAIL works by leveraging machine learning and other

19 computational approaches to identify DNA sequence features that confer cell type-specific gene

20 activation and using them to make a probe that drives an affinity purification-compatible reporter gene.

21 As a proof of concept, we designed and validated two novel SNAIL probes that target parvalbumin-

22 expressing (PV) neurons. Furthermore, we show that nuclear isolation using SNAIL in wild type mice is

23 sufficient to capture characteristic open chromatin features of PV neurons in the cortex, striatum, and

24 external globus pallidus. Expansion of this technology has broad applications in cell type-specific

25 observation, manipulation, and therapeutics across species and disease models. 


\section{Introduction}

The biology of the brain is complicated by vast diversity in cell types, subtypes, and cell states.

29 Contemporary advancements in single cell sequencing have identified over a hundred molecularly distinct neuron populations in the mammalian cortex (Hodge et al., 2019; Lake et al., 2016; Saunders et al., 2018; Tasic et al., 2018; Zeisel et al., 2015) including several small subpopulations of Gamma aminobutyric acid (GABA)ergic neurons whose specialized functions are critical for the control of neuronal inhibition (Kepecs and Fishell, 2014; Lim et al., 2018). Understanding neurological function in health and disease from a cell type-specific perspective is critical to the progress of neuroscience. manipulation of discrete cell populations. Transgenic mouse strains targeting major inhibitory neuron

37 subclasses including Parvalbumin-expressing (PV), Somatostatin-expressing (SST), and serotonergic (5HT) neurons are widely used today and have been instrumental toward our understanding of these cell types (Madisen et al., 2010; Taniguchi et al., 2011). Additional cell type-specific transgenic strains have been created through strategies like enhancer trap (Shima et al., 2016) and EDGE (Nair et al., 2020),

41 which leverage the specificity of cis regulatory sequence activity and improve the throughput of

42 transgenic development. Yet even with these innovations, as the number of cell populations of interest

43 rapidly expands, new transgenic strains cannot scale accordingly.

45 (Dimidschstein et al., 2016; Graybuck et al., 2021; Hrvatin et al., 2019; Mich et al., 2021; Nair et al.,

46 2020; Vormstein-Schneider et al., 2020). Adeno-associated virus (AAV) technologies became particularly

47 attractive with the invention of AAV variants that cross the blood-brain barrier to transduce the central

48 nervous system, AAV-PHP.B and AAV-PHP.eB (Chan et al., 2017; Deverman et al., 2016). In line with

49 certain transgenic engineering, an emerging AAV targeting strategy is to incorporate cell type-specific

50 enhancer elements into the viral genome to promote restricted expression. Enhancer activity can be

51 extremely selective, even more so than the activity of most genes and their associated promoters

52 (Hoffman et al., 2013; Kellis et al., 2014; Roadmap Epigenomics Consortium et al., 2015). Thus, 
53 enhancers may be used to confer specificity even for neuron subtypes that cannot be resolved by the

54 expression of a single marker gene (Tasic et al., 2018) or where the marker gene promoter is not specific

55 on its own (Nathanson et al., 2009).

Despite the enthusiasm for enhancer sequences in cell type-specific AAV development, their

57 selection remains nontrivial. ATAC-seq (Buenrostro et al., 2013) has been a popular technique for

58 defining potential cell type-specific enhancer regions because of its high resolution and its compatibility

59 with small cell populations and even single cell technologies (Buenrostro et al., 2015b; Cusanovich et al.,

60 2015). The biggest outstanding barrier to sequence engineering for targeted technologies is the low

61 conversion rate from experimentally suggested cell type-specific open chromatin regions (OCRs) to

62 desired cell type-specific activity in the isolated viral context. Simple enhancer sequence prioritization

63 methods using ATAC-seq signal strength or sequence conservation have been insufficient. Recently, a

64 parallel screening approach involving single nucleus sequencing of barcoded enhancer libraries, PESCA,

65 was proposed to speed up the selection process toward a successful enhancer-driven virus (Hrvatin et al.,

66 2019). Another approach leveraged cell population marker gene proximity for enhancer prioritization

67 (Vormstein-Schneider et al., 2020). We hypothesized that there were additional in silico filters that could

68 be applied to reduce the burden of experimental screening in cell type-specific AAV development.

Toward this goal, we sought to leverage the complex combinatorial code linking transcription

70 factor binding site motifs and other DNA sequence features to cell type-specific regulatory activity (Jindal

71 and Farley, 2021). To learn that code, we turned to machine learning models, which have achieved state-

72 of-the-art performance on predicting regulatory activity from DNA sequence (Ghandi et al., 2014; Kelley

73 et al., 2016; Quang and Xie, 2016). Convolutional neural networks (CNNs) (Cun et al., 1989) and support

74 vector machines (SVMs), for example, have been applied to predict enhancer activity from sequence

75 across tissues and cell types (Chen et al., 2018; Kaplow et al., 2020; Kelley, 2020). We reasoned that

76 machine learning classifiers could be applied to identify the most characteristic enhancer sequence

77 patterns within a given cell type, enabling us to prioritize and interpret sequences that are most likely to

78 drive selective expression. 
We developed a framework for machine learning-assisted engineering of cell type-specific

80 AAVs, which we refer to as Specific Nuclear Anchored Independent Labeling (SNAIL). Building upon

81 our previously described Cre-activated AAV technology cSNAIL (Lawler et al., 2020), SNAIL probes

82 have the unique advantage of expressing an affinity purification-compatible fluorescent tag (Deal and

83 Henikoff, 2010; Mo et al., 2015). This protein, Sun1GFP, enables nuclei isolation that is particularly

84 advantageous for accessing rare cell populations that would otherwise have low representation in bulk

85 tissue or single nucleus sequencing. Unlike cSNAIL, SNAIL probes are not Cre-dependent, but are

86 instead driven by cell type-specific enhancer sequences selected through machine learning models.

87 Here, we describe two novel AAV probes for PV neurons. In the mouse cortex, PV SNAIL

88 probes labeled PV neurons with $>70 \%$ specificity to Pvalb antibody staining. Isolated populations of

89 tagged cells from the cortex, striatum, and external globus pallidus (GPe) were heavily enriched for

90 known PV open chromatin signatures. In the cortex, PV SNAIL probes were more specific to GABAergic

91 PV interneurons than the common Pvalb-2A-Cre mouse strain. Nucleotide-resolution model interpretation

92 highlighted a collection of 14 transcription factor binding motif families responsible for PV neuron-

93 specific enhancer activation. These results demonstrate concrete utility in sequence-level information for

94 AAV enhancer selection, setting the stage for efficient probe design for a wide range of cell types.

\section{Results}

97 Support vector machines discriminate known cell type-specific regulatory sequences

We sought to build machine learning classifiers that could discriminate sequences of differential

99 OCRs between two cell populations. We imposed upfront that training sequences have a minimum fold

100 difference in chromatin accessibility between the cell types to ensure that the model learned cell type-

101 specific features of enhancer activation and not general enhancer features. We chose this strategy because

102 it was most closely aligned with our goal of prioritizing sequences that would activate in one cell type and 103 not others. 

classifiers, we first built SVMs comparing select broad classes of cell types in the brain. These were i) a neuron vs. astrocyte classifier and ii) an excitatory neuron vs. inhibitory neuron classifier. The training and validation sequences were based on differential OCRs between cell types, identified from single nucleus (sn)ATAC-seq data from the mouse motor cortex (MOp) (Li et al., 2020) (Supplemental Fig. 1). Both models performed well on held out data, achieving areas under receiver operating characteristic curves (auROCs) of 0.95 and 0.93 (Supplemental Fig. 2).

Next, we verified that these models could recapitulate known cell type-specific activation patterns

112 of commonly used AAV promoter sequences Gfap, CamkII, and Dlx (Supplemental Fig. 2). The Gfap

113 promoter sequence, which empirically has a heavy astrocyte bias in vivo, scored highly astrocyte-specific

114 in the neuron vs. astrocyte model, achieving a threshold with less than a $2.1 \%$ false positive rate among

115 validation data. In the same neuron vs. astrocyte model, the CamkII promoter and Dlx promoter sequences scored highly neuron-specific. Also consistent with empirical expectations, the excitatory vs.

117 inhibitory neuron model predicted the CamkII sequence to have excitatory neuron preference and the Dlx 118 sequence to have inhibitory neuron preference, while the Gfap promoter scored close to neutral 119 (Supplemental Fig. 2). Therefore, this classification strategy is capable of correctly predicting cell typespecific regulatory sequence activity in the viral context, at least for very distinct cell classes.

Machine learning models accurately predict PV neuron-specific open chromatin from sequence

Next, we assessed whether the same principles could be applied to more narrowly defined neuron

124 subtypes, using PV neurons as a target. To define potential PV neuron and PV- cell enhancer candidates

125 in the mouse cortex in a data-driven manner, we conducted ATAC-seq on the PV and PV- nuclei

126 populations of Pvalb-2A-Cre mice. The nuclei populations were isolated using previously described Cre-

127 dependent AAV affinity purification technology, cSNAIL (Lawler et al., 2020). cSNAIL probes activate

128 an isolatable nuclear envelope tag in the presence of Cre recombinase protein. Therefore, purified

129 populations from these mice are a direct reflection of cells labeled by the Pvalb-2A-Cre mouse strain, a 
current standard for PV neuron labeling. These cSNAIL PV and PV- ATAC-seq signatures ultimately defined the training data for models for designing PV SNAIL probes, which are independently activated by PV-specific regulatory elements. identified significantly differentially accessible OCRs between the two cell populations (DESeq2 padj < $0.01 \& \mid \log 2$ FoldChange $\mid>1$ ) (Love et al., 2014). To refine these regions for model training, we eliminated promoter-proximal OCRs within 2,000 base pairs (bp) of an annotated transcription start site

137 (TSS). This decision biased training examples toward OCRs of potential enhancer function, which are most relevant for cell type-specific AAV design and may have different sequence composition than gene promoters. This resulted in 14,059 PV OCRs and 4,935 PV- OCRs of interest genome-wide.

We developed two SVMs to distinguish between PV and PV- OCR classes based on nucleotide sequence, one linear model and one nonlinear model. Both SVMs were based on gapped k-mer count vectors, i.e. the number of occurrences of all short subsequences of length $\mathrm{k}$, tolerating some gaps or mismatches, as implemented by LS-GKM (Ghandi et al., 2014; Lee, 2016). The training data were 500 bp

144 sequences underlying PV or PV- OCRs of interest, with a 2.55:1 ratio of positives to negatives. The sequences were centered on ATAC-seq peak summits, where functional transcription factor binding motifs tend to be concentrated (Buenrostro et al., 2013). Taking advantage of this property, we used a

147 center-weighted kernel function for both SVMs, meaning gapped k-mers near the sequence center were

148 weighted more heavily than peripheral gapped k-mers. The two SVMs differed in that one was linear and 149 the other implemented a radial basis function (rbf) kernel, which permits the detection of interactions 150 between gapped k-mers. Both SVMs could predict the correct classification on held out data with high 151 accuracy (Fig. 1b,c), indicating that there were substantial sequence pattern differences between the PV 152 and PV- classes and that the models were able to learn these differences. to neurons, we considered the possibility that the PV vs. PV- models were learning features of general

155 neuron vs. glia enhancer sequence properties and not necessarily features that were specific to PV 
neurons. To address this issue, we trained additional population-derived SVMs that directly discriminated between enhancer sequences of PV neurons and other neuron subtypes, using publicly available ATACseq data from INTACT-sorted excitatory (EXC) neurons and VIP neurons (Mo et al., 2015). The model training data were defined with the same process described for the PV vs. PV-models. The PV vs. EXC models were trained on 27,879 PV sequence examples and 30,728 EXC sequence examples. The PV vs. VIP models were trained on 15,474 PV sequence examples and 28,683 VIP sequence examples. These models performed well (Fig. 1b,c), indicating that even at the level of neuron subtypes, OCR sequence information is rich enough to reliably distinguish cell type-specific activity.

To survey an additional machine learning strategy, we also built CNN classifiers from the same underlying data, using a different approach (Supplemental Fig. 3). CNNs are best equipped to automatically learn higher-order interactions between sequence features without explicit handcrafting of features. To define the training data for the CNNs, we binned the genome into $200 \mathrm{bp}$ bins and identified bins with differential chromatin accessibility $(q<0.01)$ between cell types. These sequences were extended bidirectionally to $1,000 \mathrm{bp}$ and used for model training and evaluation. The PV vs. PV- CNN was trained on 55,398 PV sequences and 37,919 PV- sequences, the PV vs. EXC CNN was trained on

171 3,212 PV sequences and 36,509 EXC sequences, and the PV vs. VIP CNN was trained on 22,416 PV

172 sequences and 96,609 VIP sequences. The CNNs were highly accurate (Fig. 1d), demonstrating an 173 additional approach to discriminate OCR sequence differences between purified neuron populations. examples of differentially accessible reads between neuron subtypes, many neuron populations of interest are not yet isolatable, even through transgenic means. Single nucleus sequencing technologies can be

177 applied to measure neuron subtype-resolution open chromatin without cell sorting by performing several 178 parallel micro-reactions that introduce unique cell barcodes into ATAC-seq sequencing reads. Therefore, 179 we explored whether cell type-specific enhancer sequences derived from mouse motor cortex snATAC180 seq (Li et al., 2020) were sufficient to produce neuron subtype-level classifiers. We trained several 181 pairwise linear center-weighted gapped k-mer SVMs to discriminate differential open chromatin 
sequences from snATAC-seq clusters or groups of clusters. These included analogous models to the population-derived models comparing PV vs. PV-, PV vs. EXC, and PV vs. VIP. In this case, the single nucleus-derived PV vs. PV- model refers to a model trained on differential OCR sequences comparing PV cluster nuclei to all other nuclei with a random sampling probability. The PV vs. k-nearest-neighbor $(\mathrm{KNN})$ model is an additional variation on the PV vs. PV- model where the PV-nuclei sampling for differential OCR analysis was selected for similarity to the PV cluster as implemented in SnapATAC (Fang et al., 2021). We also produced a model comparing PV vs. SST neurons, the most similar subtype to PV. The number of training examples per class of these models ranged from 13,040 to 95,694 and the positive (PV) to negative ratios per model ranged from 1:1.04 to 1:3.74 (further information available in Supplemental Table 2). Single nucleus-derived SVMs were able to classify cell type-specific enhancer sequences with high accuracy (Fig. 1e). contributions for equivalent tasks. When scoring the population-derived sequences through both the population-derived SVMs and the single nucleus-derived SVMs, individual sequences scored highly similarly in both models (Fig. 1f). These findings highlight the prevalence of reliable cell type-specific

197 enhancer sequence signatures that can be defined by a variety of classifier types and sources of open

198 chromatin measurements. The parameter and performance details of all models can be found in Tables S2 199 (SVMs) and S3 (CNNs).

\section{Models learn biological signatures relevant for AAV probe design}

We have shown that multiple machine learning strategies are useful for discriminating between regulatory sequences that are differentially active between neuron populations. Next, we asked whether

204 these models could be useful for prioritizing enhancer sequence candidates for cell type-specific enhancer

205 driven technologies. The strength of chromatin accessibility signal at an individual locus may be dynamic

206 and insufficient for cell type-specific enhancer prioritization on its own. Enhancer candidates with highly 
specific chromatin accessibility and with high specificity scores in the models represent the most characteristic cell type-specific sequence features and may be more effective than other OCRs.

First, we wanted to ensure that the success of the classifiers was rooted in biological sequence signatures related to transcription factor binding motifs. We employed GkmExplain (Shrikumar et al.,

211 2019) and TF-MoDISco (Shrikumar et al., 2018) model interpretation methods to identify sequence

212 patterns with high contributions toward PV neuron-specific OCR predictions, focusing on the population-

213 derived linear SVMs. The models learned sequence patterns that matched known transcription factor

214 binding motifs (Gupta et al., 2007). These included critical developmental transcription factors (TFs) that

215 promote PV interneuron lineage specification Lhx6, Maf, and Mef2c (Liodis et al., 2007; Pai et al., 2020;

216 Vogt et al., 2014) (Fig. 1g). This was encouraging for biological relevance, especially given that the

217 models had no knowledge of known motifs or even the concept of transcription factor binding a priori.

219 the specific purpose of creating selective PV neuron viruses, we evaluated model predictions on

220 externally validated successful and unsuccessful PV probe enhancer candidates from Vormstein-

221 Schneider et al., 2020, named E1 - E34. Importantly, the enhancer sequence from the probe with the

222 lowest PV specificity (E4; 14\% specificity) received a negative score from every model, and two probe

223 enhancers with highest cortical PV specificity (E22 \& E29; 94\% specificity) received high positive scores

224 from every model.

The average score across all models was predictive of probe specificity (Pearson correlation coefficient $=0.42, p=0.016$ ). Individual enhancer candidates tended to receive similar scores across the

227 SVMs comparing PV to highly abundant cell populations (PV vs. PV-, PV vs. EXC, PV vs. KNN), with 228 Pearson correlations between pairs of models ranging from 0.56 to 0.99 (Supplemental Fig. 4). Many of 229 these models were weakly significant predictors of empirical PV specificity in the AAV context on their 230 own, with the population-derived PV vs. EXC models reaching the highest significance $(\operatorname{padj}=0.047)$

231 (Supplemental Fig. 5). Some models, such as PV vs. KNN, were better predictors of PV probe specificity 232 than the log fold difference of chromatin accessibility for that cell comparison (Supplemental Fig. 5). 
SVMs comparing PV against rarer subtypes (PV vs. VIP, PV vs. SST) were more unique and had less correlation with other models. These models were not significant predictors of probe specificity overall, but many of the highest performing probes had positive scores. Probe specificity was not associated with PhyloP score, which has been considered in cell type-specific enhancer prioritization (Hrvatin et al.,

237 2019), but did show a trend with activity conservation at orthologous regions in the human genome

238 (Supplemental Fig. 5). Importantly, neither method of conservation was as predictive of AAV specificity 239 as the average model score.

241 drastically reduce in vivo screening efforts by signaling the best PV enhancer sequences before

242 experimentation. The models predicted which PV enhancer sequence candidates were likely to be cell

243 type-specific drivers and precisely which subsequences were responsible for PV neuron-specific

244 activation. Sequence E29, within the Inpp5j locus, was predicted to have PV neuron-specific activity due

245 to a central Mef2 motif site and nearby Err3 motif site, among others (Supplemental Fig. 6). Sequence

246 E22, within the Tmem132c locus, was predicted to have PV specificity in part due to Nkx28 and Lhx6

247 motif sites (Supplemental Fig. 6). Yet, none of these enhancers were our highest predicted PV neuron

248 sequences, so we continued to investigate additional enhancer candidates genome-wide for PV SNAIL

249 probe implementation.

251 Two candidate PV SNAIL probes successfully target PV neurons in the mouse cortex

Based on the predictions of all PV enhancer models on our candidates, we prioritized two highly

253 characteristic PV neuron enhancer sequences to test for their ability to drive targeted expression in vivo

254 (Fig. 2). We refer to these sequence candidates as SC1 and SC2. Among true PV neuron-specific

255 enhancer sequences that i) were differential OCRs in PV vs. PV-, PV vs. EXC, and PV vs. VIP sorted

256 population data and ii) scored PV positive across all SVM evaluations (1,755 sequences), SC1 was the

257 highest predicted sequence candidate, while SC2 was in the 90th percentile (Fig. 2b, Supplemental Table

258 4). 
SC1 and SC2 sequences were cloned into separate vectors upstream of the cSNAIL reporter gene, Sun1GFP. To minimize off-target effects, PV SNAIL probes directly rely on transcriptional activation from SC1 or SC2, with no minimal promoter (see methods). We also prepared two control vectors: a negative control that was the identical vector but with no inserted enhancer sequence and a nonspecific control that was the identical vector but with a common Ef1a promoter sequence in place of the candidate sequence. When packaged with AAV-PHP.eB and delivered to the mouse through systemic injection, the SC1-Sun1GFP and SC2-Sun1GFP constructs promoted cortical fluorescence that was restricted to PV neurons, while the Ef1a virus did not (Fig. 2c-e, Supplemental Table 5). Compared with immunohistochemistry-label Pvalb protein, SC1 and SC2-mediated expression of Sun1GFP was restricted to Pvalb+ neurons in $\sim 70-74 \%$ of cases. This was an 11 -fold enrichment in precision over the Ef1a promoter and notably, an almost 2-fold enrichment over Cre reporter labeling in Pvalb-2A-Cre mice. We expect these to be conservative estimates of PV targeting due to incomplete antibody capture. On average,

271 Sun1GFP expression from SC1 and SC2 SNAIL probes labeled 71-73\% of Pvalb+ neurons. The rate is

272 limited by the transduction properties of the AAV-PHP.eB capsid, which only transduces 55-70\% of

273 neurons in the cortex (Chan et al., 2017). SC1 and SC2 expression in Pvalb+ neurons represents at least a

2749 -fold increase over the negative control virus.

278 technology. The stable nuclear envelope association of this tag enables affinity purification using

279 magnetic beads coated with anti-GFP antibody, which is advantageous for rare population isolation and

280 downstream epigenetic assays. In many contexts, purification of a cell population is more efficient than

281 single nucleus sequencing technologies, especially if the population of interest is in low proportion or the

282 desired downstream applications are not available in single nucleus approaches. Taking advantage of this

283 property, we isolated Sun1GFP-expressing nuclei induced by SC1-Sun1GFP, SC2-Sun1GFP, or Ef1a-

284 Sun1GFP SNAIL virus from the mouse cortex and performed ATAC-seq. Through comparison with 
known PV neuron ATAC-seq (via cSNAIL in the Pvalb-2A-Cre strain) and PV- or bulk ATAC-seq including cSNAIL PV- cell fractions and Ef1a virus signatures, we determined that both SC1-Sun1GFP and SC2-Sun1GFP cells are highly enriched for PV neurons. neuron samples from PV- and bulk tissue samples. Likewise, SC1-Sun1GFP and SC2-Sun1GFP samples grouped with the PV samples while Ef1a-Sun1GFP samples grouped with the PV-and bulk sample signatures (Fig. 3a). At the Pvalb locus, there were highly reproducible OCR signals between PV cSNAIL, PV snATAC-seq, SC1-Sun1GFP, and SC2-Sun1GFP samples that did not appear in bulk tissue, 293 PV-, or Ef1a-Sun1GFP samples (Fig. 3b). technologies in certain contexts. Ideally then, ATAC-seq from Sun1GFP-sorted cells from SNAIL probes in wild type mice should provide similar information as ATAC-seq from Sun1GFP-sorted cells from cSNAIL in Pvalb-2A-Cre transgenic mice. Therefore, we defined PV cSNAIL ATAC-seq $\log 2$ FoldDifference over bulk cortical tissue ATAC-seq as a gold standard for each OCR. For SC1 and SC2, we computed the correlations between the log2FoldDifference of OCR signal relative to bulk tissue and the $\log 2$ FoldDifference of OCR signal in PV cSNAIL relative to bulk tissue. To establish an upper

301 limit for correlation, we compared two different batches of cortical PV cSNAIL samples, which had a 302 Pearson correlation of 0.86 and a Spearman correlation of 0.85 . As a lower limit, we evaluated the non303 specific Ef1a control virus, which had a Pearson correlation of 0.38 and a Spearman correlation of 0.26. 304 Because the AAV-PHP.eB capsid has a neuron bias, these lowly-correlated signatures are likely to be 305 general neuron specifications shared among PV and other neurons. Within this range, SC1 and SC2 had 306 very high correlation with cSNAIL, with SC1 achieving almost equivalent correlation as the two cSNAIL 307 batches $(\mathrm{SC} 1$ Pearson $=0.85$ and Spearman $=0.84 ; \mathrm{SC} 2$ Pearson $=0.81$ and Spearman $=0.79)($ Fig. $3 \mathrm{c})$. 308 The details for differential OCRs in each virus relative to bulk tissue can be found in Supplemental Table 3096. 
311 those of snATAC-seq clusters from the mouse motor cortex (Fig. 3d) (Li et al., 2020). We defined

312 cluster-specific OCRs for each snATAC-seq cluster and population-enriched OCRs for SNAIL-isolated

313 cells relative to bulk tissue (see methods) and assessed the overlaps. We found that cSNAIL-isolated PV

314 OCRs, SC1-isolated OCRs, and SC2-isolated OCRs were each significantly enriched for PV cluster-

315 specific markers ( $34 \%$ - 47\% overlap, hypergeometric $\mathrm{p}=0)$, while OCRs from Ef1a-isolated cells were

316 not enriched for PV cluster-specific markers (4\% overlap, $p=1)$. Ef1a OCRs instead had the highest

317 enrichment for markers of a layer 4 excitatory neuron cluster $\left(25 \%\right.$ overlap, $\left.p=5.3 \times 10^{-5}\right)$. We also note

318 that cSNAIL PV ATAC-seq had an additional 8\% overlap with excitatory cluster L5 PT markers $(\mathrm{p}=2.5$

$319 \times 10^{-45}$ ), possibly reflective of Pvalb-2A-Cre line labeling in layer 5 Parvalbumin-expressing excitatory

320 neurons (Jinno and Kosaka, 2004; Roccaro-Waldmeyer et al., 2018; Tanahira et al., 2009). These OCRs

321 were absent in SC1 - and SC2-isolated cells. In fact, SC1 and SC2 had no enrichment for cluster-specific

322 OCRs of any cluster other than PV ( $\leq 2 \%$ overlap, $p>0.1)$, including the closely related SST population.

323 This suggests that SC1 and SC2 SNAIL probes actually target a stricter subset of the cells than the Pvalb-

324 2A-Cre mouse strain, likely restricted to PV inhibitory interneurons.

Chromatin accessibility differences between $P V$ neurons in different brain regions

328 neurons. Many PV neurons throughout the brain have a common developmental origin in the medial 329 ganglionic eminence (MGE), but there are substantial OCR differences between mature PV neuron 330 populations in different brain regions. From cSNAIL-isolated PV populations in Pvalb-2A-Cre mice 331 (Lawler et al., 2020), we characterized thousands of OCRs with differential accessibility between the 332 cortex, striatum, and GPe ( $\mathrm{p}_{\text {adj }}<0.01, \mid \log 2$ FoldDifference $\left.\mid>1\right)$ (Fig. 4a, Supplemental Table 7). These

333 differences were associated with distinct TF binding motifs (Fig. 4b, Supplemental Table 8). For

334 example, OCRs that were more accessible in cortical PV neurons relative to striatal and GPe PV had

335 highest enrichment for Mef2a motifs, an activity-dependent transcription factor that is important in 
336 plasticity and distinguishes subpopulations of PV neurons in the hippocampus (Donato et al., 2015).

337 Mef2c has a similar binding motif and is the second-highest enriched TF motif in cortex-specific PV

338 neuron OCRs. Mef2c is essential for specifying the MGE PV neuron lineage in mouse and human (Mayer

339 et al., 2018) and has been linked to Schizophrenia and other neurodevelopmental disorders (Mitchell et

340 al., 2018). TFs with motifs enriched in PV neuron OCRs that are more open in striatum relative to cortex

341 and GPe included Tgif1, a key homeodomain gene involved in holoprosencephaly (Taniguchi et al.,

342 2012). At 6,654 differential OCRs, GPe-specific PV OCRs were the most unique, and had TF motif

343 enrichments including the Lhx3, Pou5f1, Err3, and Pax3 motifs.

344 These molecular differences likely relate to functional differences, for example, the tendency of

345 PV cells in the GPe to project to other brain regions versus the local nature of PV cells in the cortex

346 (Hernández et al., 2015; Saunders et al., 2016). We assessed ontology enrichments in the brain region-

347 specific PV ATAC-seq OCR sets relative to all PV ATAC-seq OCRs using GREAT (McLean et al.,

348 2010) (Supplemental Table 9). The set of PV OCRs enriched in cortical PV neurons included 10 regions

349 associated with the Bdnf gene (Ensembl Genes; FDR Q = 0.0035). Among these was Bdnf promoter IV

350 which is known to be essential for PV neuron synaptic transmission in the prefrontal cortex (Sakata et al.,

351 2009). Other cortex-specific PV enrichments included terms related to sensory perception, especially

352 smell. Striatum-specific PV neuron OCRs were enriched for the adenylate cyclase-inhibiting dopamine

353 receptor signaling pathway $(\mathrm{GO}: \mathrm{BP} ; \mathrm{FDR} \mathrm{Q}=0.010)$ and bradykinesia (Mouse Phenotype; FDR $\mathrm{Q}=$

354 0.046). OCRs preferentially open in GPe PV neurons were enriched for neuropeptide signaling pathways,

355 for example acetylcholine receptor binding (GO:MF; FDR Q = 0.0044) and neuropeptide receptor activity

356 (GO:MF; FDR Q $=1.2 \times 10^{-5}$ ). This suggests unique epigenetic mechanisms for the regulation of

357 transcription related to receptor signaling in GPe PV neurons, but further work is needed to discern these

358 relationships. 
Given these complexities, we were interested in the extent to which PV enhancer probes chosen from data in one tissue could generalize to other brain regions. Here, we assessed whether SC1 and SC2 SNAIL probes, designed in the cortex, were also selective for PV neurons in the striatum and GPe. First, we used cSNAIL ATAC-seq data from the striatum and GPe to model the regulatory sequence properties of PV neurons vs. PV- cells in these brain regions (Supplemental Fig. 7), and tested whether SC1 and SC2 sequences were predicted to have PV-specific activation (Fig 4c,f). Indeed, SC1 and SC2 were predicted to have PV neuron-specific activity in striatum and GPe PV vs. PV- SVMs. However, there were 1-3,000 sequences with more confident scores toward PV specific activity in each case. Sun1GFP affinity purification and performed ATAC-seq on the tagged populations. We have previously shown high agreement between cSNAIL and Pvalb-2A-Cre labeling in the striatum and GPe (Lawler et al., 2020), so we again used cSNAIL ATAC-seq samples from these regions as true PV neuron signals.

373 By principal component analysis (PCA), we recovered separation between PV samples, including SC1 and SC2-isolated populations, and PV- samples (Fig. 4d,g). We assessed the correlations between $\log 2$ FoldDifference in SNAIL and cSNAIL samples, each relative to bulk tissue (striatum) or, where there were no bulk samples available, cSNAIL PV- cells (GPe) (Fig. 4e,h, Supplemental Table 10,

377 Supplemental Table 11). Pearson correlation coefficients were similar or slightly lower for SC1 and SC2

378 in the striatum and GPe than for equivalent comparisons in the cortex, indicating less conservation

379 between cSNAIL and SNAIL probe targets $(\mathrm{SC} 1$ cortex $=0.85$, striatum $=0.71, \mathrm{GPe}=0.68 ; \mathrm{SC} 2$ cortex $380=0.81$, striatum $=0.82, \mathrm{GPe}=0.73)$. Yet, these were substantially increased over Ef1a correlation with 381 cSNAIL in these tissues, especially for the striatum $(\mathrm{Ef1a}$ cortex $=0.38$, striatum $=0.18, \mathrm{GPe}=0.51)$. By comparing the overlaps of SC1 and SC2-enriched OCRs in striatum and GPe with cortical snATAC-seq cluster-specific OCRs, we still identified the PV cluster as most similar to SC1 and SC2 cells. As expected, all overlaps in striatum-cortex and GPe-cortex comparisons were lower than those

385 from cortex-cortex comparisons, but the magnitudes of SC1 and SC2 overlap with the Pvalb cluster in 386 these brain regions were similar to the magnitudes of cSNAIL PV overlap with the Pvalb cluster in these 
brain regions (Supplemental Fig. 8). In the striatum, the overlaps with the Pvalb cluster were 8\% for SC1, $14 \%$ for SC2, and $14 \%$ for cSNAIL. In the GPe, the overlaps with the Pvalb cluster were $7 \%$ for SC1, 7\% for SC2, and 9\% for cSNAIL. From these interpretations, SC1 and SC2 SNAIL viruses do generalize to the striatum and GPe, though they may not be as robust as they are within the cortical context.

Err3 and Mef2 motifs are important for the PV-specific activity of SC1 and SC2 sequences neuron-specific activity prediction, we assessed commonly used motifs for each model and identified potential matches within the candidate sequences. For all SVMs, we calculated per-base importance scores and hypothetical importance scores for the set of PV-specific OCRs that were true positives according to all SVMs (score $>0 ; \mathrm{N}=1,755$ ) (Shrikumar et al., 2019). Then, for each model, we used TF-MoDISco (Shrikumar et al., 2018) to cluster commonly important subsequences called "seqlets" within these PV-specific examples. The resulting clusters represent motifs that were high contributors to a positive score in each model. Among the 11 SVMs comparing PV neuron open-chromatin against PVappeared to be shared across multiple models. Thus, we performed UPGMA clustering on the 124 motifs by sequence similarity using STAMP (Mahony and Benos, 2007) and identified 14 motif clusters (Fig.

The largest cluster, with 23 motif members, contained representation from all 11 models and had matches to known motifs including the motifs for Err3 and Rora (Supplemental Table 12). Consistent 407 with an important role for Err3 in PV neurons, Err3 (a.k.a. Esrrg) transcript levels were differentially 408 over-expressed in the PV neuron cluster relative the rest of the frontal cortex in snRNA-seq (DropViz 409 subcluster \#2-7 Neuron.Gad1Gad2.Pvalb Esrrg fold ratio = 8.0, p = 1.14 x 10-198) (Saunders et al., 410 2018). Esrrg and Rora are key TFs in the Pgc1a transcriptional program, which regulates Pvalb 411 expression, mitochondrial function, and transmitter release (Lin et al., 2005; Lucas et al., 2010). Pgc1a 
412 signaling is restricted to PV neurons in the brain, and may mediate the unique energy demands of fast-

413 spiking neurons (Lucas et al., 2014; Paul et al., 2017).

414 The second largest motif cluster contained 16 motifs, also representing all 11 models, and the

415 motifs had best matches to motifs for Mef2a, Mef2c, and Mef2d. In finer subdivisions of this cluster, PV

416 vs. VIP model motifs had best matches to Mef2a, while all other models tended to have best matches for

417 Mef2c and Mef2d. A cluster of Lhx6-like motifs, a transcription factor necessary for MGE interneuron

418 differentiation from interneuron progenitors (Liodis et al., 2007; Vogt et al., 2014), was detected with

419 high support from PV vs. PV- models and PV vs. EXC models, low support from PV vs. VIP models, and

420 not detected between MGE neuron subtypes PV vs. SST. Interestingly, two clusters of motifs were

421 dominated by PV vs. VIP signal, including matches for Stat6, Nkx28, and Cux2 motifs. Cux2 expression

422 is induced by Lhx6 in the MGE, supporting a role in specification of the MGE interneuron lineage

423 (including PV and SST neurons) from other interneuron lineages (Zhao et al., 2008). Overall, these

424 findings indicate both shared and unique sequence properties dictating PV-specific regulatory sequence

425 activity relative to other cell types.

SC1 and SC2 represent two experimentally validated PV-selective regulatory sequences. To

427 interpret the sequence determinants of their success, we mapped potential motif sites for the $124 \mathrm{TF}$ -

428 MoDISco motifs (Supplemental Table 13) and overlaid these with per-base importance scores for each of

429 the SVMs (Supplemental Table 14). This strategy revealed multiple high importance subsequences with

430 potential transcription factor binding function. SC1 contained two Err3 motifs near the sequence center

431 which were high contributors to the PV-specific model predictions and matched TF-MoDISco motifs for

432 every model (Fig. 5b). An additional subsequence with contributions specific to PV vs. VIP models

433 matched motifs for Sp7. SC2 contained a highly important Mef2 sequence near the center (Fig. 5c). This

434 was a specific match for Mef2c and Mef2d motifs and excluded Mef2a motifs from PV vs. VIP models.

435 Additionally, SC2 contained an Err3 motif with shared importance across all models. Interestingly, the

436 most important features of the SC2 sequence closely resemble those of successful PV probe E29 from

437 Vormstein-Schneider et al., 2020 (Vormstein-Schneider et al., 2020) (Supplemental Fig. 6). The success 
of SC1 and SC2 are both largely explainable by transcription factor binding motif properties and represent two sequence pattern strategies toward PV-specific activation.

\section{Discussion}

OCR sequence features provide valuable, underutilized information for cell type-specific

443 enhancer design. Here, we showed that sequence alone was sufficient to discriminate between OCR

444 activity in different neuron subtypes. Interpretation of these models revealed rich diversity among the

445 biochemical underpinnings of these classification tasks, reflective of cis-trans interactions. The defining

446 sequence properties of cell type-specific OCR activation were robust throughout different data modalities,

447 including ATAC-seq from sorted populations and snATAC-seq, and different classifier types. Machine

448 learning and computational methods, broadly, can facilitate prioritization of AAV enhancer candidates by

449 quantifying sequence properties that are most characteristic and specific to a given cell type.

452 PV enhancer-driven AAVs (Vormstein-Schneider et al., 2020), the average PV-specificity score across 11

453 classifiers was more predictive of PV-specific AAV expression than the $\log 2$ fold difference of snATAC-

454 seq signal, sequence conservation, or activity conservation at these loci. With the SNAIL framework, we

455 identified and validated two novel enhancers that drive targeted expression in PV neurons in the mouse

456 cortex. While these do not represent enough trials to establish a new conversion rate from cell type-

457 specific OCRs to cell type-specific AAVs, we were encouraged by the immediate success of the first

458 probes we selected. We believe that incorporation of differential sequence property analyses like those

459 used here will continue to improve the throughput of targeted AAV development in new contexts.

460 An additional advantage of incorporating classifiers for cell type-specific enhancer selection is

461 increased interpretability of the factors that govern success. The sequence patterns learned by PV models

462 reflected known PV neuron biology. Common motifs contributing to successful PV probe enhancers

463 included Err3, Mef2, and Lhx6, important in the specification and maintenance of the cortical PV 
interneuron lineage (Liodis et al., 2007; Mayer et al., 2018; Zhao et al., 2008). SC1 and SC2 depend particularly on Mef2 and Err3 motifs for PV specificity.

$$
\text { We found that a combination of multiple direct comparisons between the target cell type and }
$$
other cell types made for particularly useful screening. Here, we used a tiered approach to ensure specific activity at multiple levels of cellular relationships to PV neurons. At the broadest level, we modeled PV neuron OCR sequences against PV- OCRs, a mixed signature from all other neuron and non-neuron cell types in the mouse cortex. Within neurons, we modeled PV vs. EXC neurons, and then PV relative to

471 more specific subtypes of inhibitory neurons VIP and SST. Successful SC1 and SC2 sequences contained

472 attributes that made them highly PV specific across all of these comparisons.

474 isolation in diverse systems. A unique feature of these viruses is the modified Sun1GFP tag that enables

475 nuclei purification by magnetic beads coated with anti-GFP antibody. This process is advantageous for

476 isolating genomic and epigenomic signals from the population of interest with no dependence on

477 transgenic strains. In comparison to single nucleus sequencing technologies, affinity purification with

478 SNAIL is more efficient for addressing targeted hypotheses about a specific cell type. SNAIL may also be

479 paired with single nucleus sequencing technologies for unprecedented resolution of the substructures

480 within minority cell populations. We took advantage of SNAIL affinity purification to isolate SC1-

481 Sun1GFP and SC2-Sun1GFP nuclei for molecular assessment with ATAC-seq. This represents a novel

482 approach for validating new cell type-specific AAVs. We found that SC1 and SC2 PV SNAIL probes had

483 high molecular agreement with cells tagged in the Pvalb-2A-Cre mouse strain, making them a reasonable

484 alternative to transgenic strain technology. In addition to their success in the intended brain region

485 (cortex), these SC1 and SC2 PV SNAIL viruses also generalized to subcortical regions, the striatum and

486 GPe.

In general, pairing cell type-specific enhancers with AAVs provide much more flexibility and

488 scalability than transgenic technologies. However, there are drawbacks in certain applications. AAVs

489 require time to reach peak expression, usually 2-4 weeks, although some may be robust earlier. This 
means they are not appropriate for developmental studies in very young animals. Additionally, there are

491 limitations to the transduction efficiency, so AAVs may not be ideal for studies where it is important to

492 label all cells of a certain type. Finally, enhancer activity in AAVs may fluctuate under different ages or

493 in response to different conditions, because enhancers are dynamic actors in the regulation of gene

494 expression. However, machine learning model-based prioritization of characteristic sequences may

495 minimize this risk.

496 Excitingly, there are many opportunities for extensions of the SNAIL framework that enable cell

497 type-specific interrogation in unprecedented settings. Machine learning model-selected enhancer

498 sequences may be used to drive the expression of a gene for cell type-specific circuit manipulation, as has

499 been achieved with channelrhodopsin and DREADDS (Lee et al., 2010; Vormstein-Schneider et al.,

500 2020). Other important advancements could overexpress a particular ion channel, neurotransmitter

501 receptor, gene variant, or guide RNA for a CRISPR-based gene manipulation strategy. More so than other

502 strategies for cell type-specific AAV design, the SNAIL framework can be tuned for cross-species probe

503 development. In fact, multiple machine learning models have successfully predicted enhancers across

504 mammals, demonstrating high evolutionary conservation in the rules for enhancer sequence activity

505 (Chen et al., 2018; Kaplow et al., 2020; Kelley, 2020; Minnoye et al., 2020). Multispecies models could

506 further improve transferability of probes across species. A new approach that explicitly encourages the

507 model not to learn signatures of species-specific enhancer activity might be especially promising

508 (Cochran et al., 2021). Lastly, while most previous enhancer selection has relied on sorted populations of

509 nuclei from existing transgenic animals, the SNAIL framework provides the opportunity to develop viral

510 tools targeting previously unexplored cell types that are identifiable in snATAC-seq. There is potential to

511 divide subpopulations at multiple levels and design extremely specific technologies. Other applications

512 may exploit changes in enhancer sequence activity in disease and other contexts to target specific cell

513 states. Continued exploration at the intersection of machine learning and enhancer technology

514 development is sure to enhance the impending era of cell type-specific neuroscience and further our

515 general understanding of specific cell types throughout the body. 


\section{Materials and Methods}

$$
\text { Experimental design. The initial cSNAIL experiments to define candidate PV enhancers were }
$$
performed on primary motor cortex and isocortex samples in triplicate on female mice aged 2-3 months old. All subsequent cSNAIL and SNAIL molecular experiments for the validation of PV SNAIL probes were performed in the cortex, striatum, and GPe with two or three biological replicates. Each of these

522 cohorts included at least one male and one female mouse, all 2-4 months old. Control samples for SNAIL

523 comparisons included cSNAIL PV, cSNAIL PV-, and cells labeled by the Ef1a-Sun1GFP virus. Details

524 for all experiment samples can be found in Supplemental Table 1. Data primary to this publication can be

525 accessed through the NCBI Gene Expression Omnibus (https://www.ncbi.nlm.nih.gov/geo/), accession

526 number GSE171549.

Nuclei isolation for ATAC-seq. ATAC-seq data were generated using an affinity purification approach with cSNAIL or SNAIL to isolate PV neurons from the mouse isocortex, as described in Lawler et al., 2020. Briefly, mice were overdosed with isoflurane, decapitated, and rapidly dissected. Fresh brain tissue was sectioned coronally on a vibratome for precision, and we dissected brain regions relevant to the 531 specific experiment to be processed as separate samples. All dissections took place in cold, oxygenated 532 artificial cerebrospinal fluid (aCSF). After dissection, we isolated nuclei from the samples by 30 strokes 533 of dounce homogenization with the loose pestle (0.005 in clearance) in lysis buffer as described in 534 Buenrostro et al., 2015 (Buenrostro et al., 2015a). The nuclei were filtered through a 70 $\mu \mathrm{m}$ strainer and 535 pelleted with 10 minutes of centrifugation at $2,000 \mathrm{xg}$ at $4{ }^{\circ} \mathrm{C}$. We resuspended the nuclei pellets in wash 536 buffer (0.25 M Sucrose, $25 \mathrm{mM} \mathrm{KCl,} 5 \mathrm{mM} \mathrm{MgCl}_{2}, 20 \mathrm{mM}$ Tricine with $\mathrm{KOH}$ to $\mathrm{pH} 7.8$, and $0.4 \%$

537 IGEPAL) for the affinity purification steps. 
542 Sun1GFP+ fraction from the Sun1GFP- fraction on a magnetic bead rack. Sun1GFP- nuclei in the

543 supernatant were centrifuged at $2000 \mathrm{x}$ g for 10 minutes to pellet nuclei, washed one time, and filtered

544 with a $40 \mu \mathrm{m}$ cell strainer. The Sun1GFP+ nuclei attached to the beads were washed 3-4 times with 800

$545 \mu \mathrm{L}$ wash buffer by resuspending the sample, letting it settle onto the magnet, and removing the buffer.

546 Where cell yield was not a concern, we also performed a large volume wash with $10 \mathrm{~mL}$ wash buffer and

547 filtered through a $20 \mu \mathrm{m}$ cell strainer. All nuclei preparations were resuspended in water for the ATAC-

548 seq reaction.

ATAC-seq library construction. For each sample, a small aliquot was stained with DAPI (Thermo

550 Fisher Scientific; cat. 62248) and the concentration of nuclei was determined by counting DAPI+ nuclei

551 with a hemocytometer. Next, we combined 50,000 nuclei, $25 \mu \mathrm{L}$ Tagment DNA Buffer, and $2.5 \mu \mathrm{L}$

552 Tagment DNA Enzyme I (Illumina, San Diego, CA; cat. 20034198) into $50 \mu \mathrm{L}$ total for the transposition

553 reaction. The reaction incubated at $37^{\circ} \mathrm{C}$ for 30 minutes with $300 \mathrm{rpm}$ mixing. Samples containing beads

554 were gently resuspended every 5-10 minutes throughout the incubation to prevent the beads from staying

555 settled at the bottom. Immediately following incubation, the DNA was column purified with the Qiagen

556 MinElute PCR Purification kit (Qiagen, Hilden Germany; cat. 28004). Libraries were amplified to 1/3

557 saturation with dual-indexed Illumina primers (Preissl et al., 2018). We ensured that samples had the

558 characteristic periodic fragment length distribution of high quality ATAC-seq using TapeStation

559 assessment (Agilent Technologies, Santa Clara, CA). Successful samples were sequenced at low depth on

560 the Illumina Miseq system to determine appropriate library pooling and sequencing depth, then paired-

561 end sequenced for $2 \times 150$ cycles with the Illumina Novaseq 6000.

Animal use. All animals for ATAC-seq experiments were either wild type mice (C57BL/6J;

563 Jackson Laboratory, Bar Harbor, ME; Stock No: 000664) for SNAIL experiments or heterozygous Pvalb-

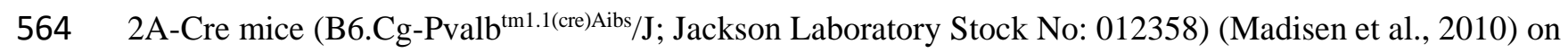

565 a C57BL/6J background for cSNAIL experiments. Imaging animals were either Pvalb-2A-Cre or double

566 transgenic Pvalb-2A-Cre/Ai14 (Ai14 strain; B6.Cg-Gt(ROSA)26Sor ${ }^{\text {tm14(CAG-tdTomato)Hze } / J ; ~ J a c k s o n ~}$

567 Laboratory Stock No: 007914). All mice were 2-4 months old at the time of the tissue experiments. Initial 
PV cSNAIL data for creating the sorted cell PV vs. PV- model was collected from female mice, but all subsequent validation experiments included representation from both sexes. All animals were housed with a 12 hour light cycle, and experiments were performed 2-3 hours after lights on. Animals for the data

571 primary to this study received no treatments other than the retro-orbital AAV injections. However,

572 previously published cSNAIL data used in analysis included healthy animals that received stereotaxic

573 saline injections to the medial forebrain bundle (Lawler et al., 2020).

Molecular cloning. To make the non-specific control viral vector pAAV-Ef1a-Sun1GFP, we

made modifications to pAAV-Ef1a-Cre with restriction enzyme cloning. pAAV-EF1a-Cre was a gift from

576 Karl Deisseroth (Addgene, Watertown, MA; plasmid \#55636; http://n2t.net/addgene:55636;

577 RRID:Addgene_55636). First, we added a multiple cloning site before the Ef1a promoter to create easy

578 promoter swapping for later use. The multiple cloning site insert was synthesized as by Integrated DNA

579 Technologies, Coralville, IA and was inserted between BshTI and MluI sites upstream of the Ef1a

580 promoter. Next, we used BamHI and EcoRI sites to replace the Cre gene with a modified Sun1GFP gene

581 identical to the one in our cSNAIL technologies.

The resulting pAAV-Ef1a-Sun1GFP vector was then further modified to create the other sequence, a synthetic intron for RNA stabilization, the Sun1GFP gene, a WPRE signal, and a polyA signal. From pAAV-Ef1a-Sun1GFP, the Ef1a promoter and intron region was removed and replaced with the sequence for a PV-specific enhancer candidate and the synthetic intron. Inserts for SC1 and SC2 were 587 synthesized by Integrated DNA technologies and cloned into the vector using restriction sites for NdeI

588 and BamHI. To ensure that no expression was being driven from the synthetic intron sequence itself, we 589 similarly cloned a negative control construct containing the synthetic intron, but no enhancer candidate 590 sequence. All transformations during cloning were performed in MegaX DH10B cells (Invitrogen,

591 \#C640003) and confirmed with Sanger sequencing.

592 AAV production. AAV was produced in AAVpro(R) 293T cells (Takara, Kyoto, Japan; \#632273)

593 by co-transfection of the genome pAAV, an AAV helper plasmid, and pUCmini-iCAP-PHP.eB. 
594 pUCmini-iCAP-PHP.eB was a gift from Viviana Gradinaru (http://n2t.net/addgene:103005; RRID:

595 Addgene 103005) (Chan et al., 2017). The AAV particles were precipitated with Polyethylene Glycol

596 (PEG 8000, Sigma-Aldrich, St. Louis, MO; cat. P2139-500G) and purified on an iodixanol gradient

597 (OptiPrep, Sigma-Aldrich, cat. D1556-250ML) with ultracentrifugation for 2.5 hours at 350,000 x g at 18

$598{ }^{\circ} \mathrm{C}$. We filtered and concentrated the virus in PBS using Amicon Ultra-15 centrifugation filters (Millipore,

599 Burlington, MA; \#UFC905024). The viral titer was measured with the AAVpro(R) Titration Kit (Takara,

$600 \# 6233)$, diluted to a concentration of $8.0 \times 10^{9}$ vector genomes $(\mathrm{vg}) / \mu \mathrm{L}$, and stored single-use aliquots at $601-80{ }^{\circ} \mathrm{C}$ until injection.

$A A V$ delivery. Animals were anesthetized with 2-3\% isoflurane until no pedal withdrawal reflex was observed. Then, we injected $4 \times 10^{11} \mathrm{vg}$ total $(50 \mu \mathrm{L})$ of virus into the retro-orbital cavity and treated the eye with $0.5 \%$ Proparacaine Hydrochloride Ophthalmic Solution. The animals were monitored while the virus incubated for 3-4 weeks until endpoint experiments. antibodies. Images were taken of the motor cortex with laser scanning confocal microscopy. Cells were counted in each channel with Fiji (Schindelin et al., 2012) and assigned as double-labeled or singlelabeled manually. Individual images from 1-3 mice were treated as replicates to determine the mean and 614 standard error of the mean for specificity and efficiency quantifications.

616 ENCODE ATAC-seq pipeline (https://github.com/ENCODE-DCC/atac-seq-pipeline) with the following

617 changes from default behaviors: atac.cap_num_peak $=300000$, atac.idr_thresh $=0.1$. All samples had

618 high TSS enrichment (>15) and clear periodicity, indicative of good data quality. Optimal IDR peaks

619 were determined for biological replicates of the same cell type, brain region, and sequencing batch 
620 (https://github.com/kundajelab/idr) (Li et al., 2011). IDR peaks were then merged to define the combined

621 peak regions (OCRs) for each analysis using bedtools (Quinlan and Hall, 2010). Specifically, we defined

622 sets of OCRs for i) cortex PV and PV-cSNAIL samples, ii) PV, EXC, and VIP INTACT samples (Mo et

623 al., 2015), and iii) cortex, striatum, and GPe bulk samples, PV and PV- cSNAIL samples, SC1-Sun1GFP

624 samples, SC2-Sun1GFP samples, and Ef1a-Sun1GFP samples. We constructed count tables including the

625 relevant samples on each of these OCR backgrounds using Rsubread featureCounts version 1.28.1 (Liao

626 et al., 2019). These three count tables were used to form the basis of i) the sorted population PV vs PV-

627 models, ii) the sorted population PV vs. EXC and PV vs. VIP models, and iii) analysis of SC1 and SC2

628 SNAIL PV probes in the cortex, striatum, and GPe.

629 The counts were modeled using the negative binomial distribution in DESeq2 (Love et al., 2014).

630 We assessed the coefficient of cell group, where cell groups were unique tissue, virus, cell type

631 combinations, and we controlled for sex differences where both were present: DESeq2 design $\sim \operatorname{sex}+$

632 cellGroup. Differential peaks were defined strictly for applications i and ii related to building models

633 (padj < 0.01 and $\mid \log 2$ FoldDifference $\mid>1$ ) and more loosely for application iii to compare across viruses

634 (padj < 0.05 and $\mid \log 2$ FoldDifference $\mid>0.5$ ). Related to Fig. 3, only cortical samples from count matrix

635 iii were included in the DESeq2 model, while the Fig. 4 DESeq2 model included samples from all three

636 brain regions.

snATAC-seq processing. The following samples of snATAC-seq from the mouse MOp were

638 downloaded in Snap file format from http://data.nemoarchive.org/biccn/: CEMBA171206_3C,

639 CEMBA171207_3C, CEMBA171212_4B, CEMBA171213_4B, CEMBA180104_4B,

640 CEMBA180409_2C, CEMBA180410_2C, CEMBA180612_5D, and CEMBA180618_4D(Li et al.,

641 2020). These were processed using SnapATAC version 1.0.0 (Fang et al., 2021). We restricted the

642 analysis to nuclei that passed filtering as defined by the original authors ( $\mathrm{Li}$ et al., 2020). This removed

643 nuclei that had at fewer than 1000 reads, TSS enrichment <10, or doublet signatures detected by Scrublet

644 (Wolock et al., 2019). Filtered samples contained 6,700-10,983 nuclei each, for a total of 78,525 nuclei.

645 We applied a bin matrix with a bin size of 5,000 and combined the snap objects. Then, we removed bins 
overlapping with the ENCODE blacklist, mitochondrial regions, and the top 5\% of bins that overlapped with invariant features. We reduced dimensionality and selected 18 significant components, then corrected for batch effects using Harmony (Korsunsky et al., 2019). We performed Louvain clustering using runCluster() with the option louvain.lib="R-igraph". genes (Supplemental Fig. 1) and by comparison to the cell annotations from the original authors (Li et al.,

652 2020). Peaks were called for each cluster using MACS2 with the options --nomodel --shift 0 --ext 73 --

653 qval 1e-2 -B --SPMR --call-summits (Zhang et al., 2008). Overlapping peaks across all clusters were

654 merged, resulting in 415,813 OCR regions in total. Differential OCRs were defined using the findDAR()

655 function with test.method = "exactTest" and were required to meet padj $<0.01$ (Benjamini-Hochberg

656 corrected) and $\mid \log 2$ FoldDifference $\mid>1$. For comparisons to groups of clusters, e.g. PV vs. EXC, separate

657 tests were performed for PV vs. each excitatory cluster, and the intersection of differential OCRs was selected.

SVM data preparation. SVMs were developed to predict the direction of differential activity from sequences underlying differential OCRs between two cell types or groups of cell types. Because ATACseq summit regions are highly enriched for transcription factor binding motifs, we centered on the peak summits within differential ATAC-seq OCRs and extended in both directions for a total fixed sequence length of $500 \mathrm{bp}$, a convenient length for AAV cloning. Peak summits were defined by MACS2 (Zhang et al., 2008), and only summit regions of peaks called within the cell type of interest were retained. For data from sorted cells, we used optimal IDR peaks across biological replicates of the given cell type. For example, in a PV vs. VIP model comparison, the positive model input examples were 500 bp summit-

667 centered regions of PV IDR peaks that overlapped PV-specific differential open chromatin regions and

668 the negative model input examples were $500 \mathrm{bp}$ summit-centered regions of VIP IDR peaks that

669 overlapped VIP-specific differential open chromatin regions. For snATAC-seq data, we used peaks called

670 within a cluster to define the relevant summit regions. If multiple cell clusters were involved in the

671 comparison, e.g. the excitatory neuron vs. inhibitory neuron model, we used summits found in any peak 
672 set from a cluster within that category. In cases where there were multiple summits within a differential

673 open chromatin region, all summits greater than $100 \mathrm{bp}$ apart from each other were retained.

674 After defining the genomic locations of the summit-centered differential open chromatin regions

675 for model training, we used additional filtering to prepare the data for model training. First, we restricted

676 the models to enhancer regions because they have more specificity than promoters and may be governed

677 by different sequence properties. Therefore, we filtered out regions that were within 2,000 bp of a TSS,

678 using RefSeq annotations downloaded from the UCSC Table browser in July 2020 (Kuhn et al., 2013).

679 Next, we removed super-enhancers because they also may be governed by different sequence features and

680 are not useful for AAV probe design because they are too large. We downloaded mm9 coordinates of

681 mouse cortex super enhancers defined by H3K27ac from the dbSuper database (Khan and Zhang, 2016)

682 and converted these to mm10 coordinates using UCSC liftOver with minmatch $=0.95$ (Kuhn et al., 2013).

683 Using bedtools intersect (Quinlan and Hall, 2010), we removed regions with any super enhancer overlap.

684 Finally, we used bedtools getfasta (Quinlan and Hall, 2010) to retrieve the sequences at these genomic

685 coordinates from the mm10 assembly, downloaded from UCSC genome browser in May 2018 (Kuhn et

686 al., 2013), and we removed any sequences that contained uncertain bases (Ns).

SVM model construction. Sequences were divided into separate partitions by chromosome for

688 model training, validation, and final testing. The training sets included chromosomes 3-7, 10-19, and X,

689 the validation sets included chromosomes 8 and 9, and the test sets included chromosomes 1 and 2. The

690 training data were input into LS-GKM's gkmtrain and evaluated with gkmpredict (Lee, 2016). Because

691 the input data was summit centered, all models used the center weighted gkm kernel, option -t 4, or the

692 center weighted gkm rbf kernel, option -t 5. The -1, -k, -d, -c, and -w parameters for word length, number

693 of informative columns, number of mismatches to consider, regularization, and class-weighted

694 regularization were tuned to maximize the validation set F1 scores through manual iterations. Other

695 parameters were left on default behavior. auROC and auPRC metrics were calculated and visualized on

696 training, validation, and test sets using the ROCR package in R (http://ipa-tys.github.io/ROCR/). All 
paper figures reflect final test set performance. The details of all parameter settings and performance metrics of the final models are reported in Supplemental Supplemental Table 2.

$$
\text { CNN data preparation. We conducted differential accessibility analysis using DESeq2 (Love et }
$$
al., 2014) to identify regulatory regions that display cell type-specific accessibility in ATAC-seq in PV neurons relative to other background cell types (PV-, VIP, EXC). We used PV and PV- neuron ATACseq samples generated in this study as well as PV, VIP, and EXC neuron ATAC-seq samples from Mo et al., 2015. To conduct differential accessibility analysis, we obtained genomic coordinates of all $200 \mathrm{bp}$ bins in the mm10 reference genome, starting from the $200 \mathrm{bp}$ bin at the beginning of each chromosome of including all following contiguous non-overlapping $200 \mathrm{bp}$ bins. We then filtered out any bin that overlaps with an artifact region (Amemiya et al., 2019) or with regions that have unknown nucleotides (obtained from the UCSC twoBitInfo utility using the -nBed option). During this step, regions near the ends of chromosomes were filtered out. Then, using the featureCounts function in the subread package (Liao et al., 2014), we counted the reads mapping to each of the $200 \mathrm{bp}$ bins in the ATAC-seq samples

711 to identify bins that were differentially accessible between i) PV and PV-, ii) PV and VIP, and iii) PV and

712 EXC neurons at a Benjamini-Hochberg FDR adjusted p-value cutoff of 0.01. For each of the three

713 comparisons, significant differential bins that displayed PV specificity (log2FoldDifference $>0$ ) were

714 used as positive examples for CNN training and significant differential bins that displayed negative

$715 \log 2$ FoldDifference ( $\log 2$ FoldDifference $<0)$ were used as negative examples for CNN training.

717 comparative regulatory activity (Kelley et al., 2016; Quang and Xie, 2016; Zhou and Troyanskaya, 2015).

718 For each significant differential $200 \mathrm{bp}$ bin, we obtained the $1000 \mathrm{bp}$ sequence surrounding the center of

719 the bin from the mm10 reference genome and trained the CNN to predict the positive or negative class

720 label. We held out sequence examples underlying all significant differential bins on chromosome 4 as a

721 validation set to evaluate hyperparameter settings and to choose the best performing final model. We also

722 held out sequence examples underlying all significant differential bins on chromosomes 8 and 9 as a test 
set for final evaluation. Because we had different validation and test sets from those used for the SVM,

724 we did not use any results from the SVM to influence our approach to designing the CNN architecture or

725 any other aspects of CNN training. We implemented our CNN model in Keras 2.2.4 (https://keras.io/)

726 with a theano backend (The Theano Development Team et al., 2016). We created a one-hot encoded

727 representation of the sequence, a $4 \times 1000$ binary matrix representing positions and occurrences of the 4

728 nucleotide characters (A,T,G and C) on the sequence, which was propagated through the network. Our

729 CNN architecture consisted of multiple layers of convolution kernels stacked on top of each other

730 (Supplemental Fig. 3). The first such layer consisted of 1000 convolution kernels, each with a kernel

731 width of 8 and height of 4 , which scan the input sequence in chunks of 8 nucleotides. We applied rectified

732 linear unit (ReLu) activations on the outputs of these convolution kernels. This initial layer is followed by

733 a variable number of convolution layers with the same number of kernels (100), each of width 8 and

734 height 1 . We applied ReLu activations on these convolution outputs as well. These convolution layers are

735 then followed by a set of max pooling operations that selects the maximum value from a set of 13

736 adjacent units (pooling size $=13$ ). We set the stride for the max pooling operation to 13 units, meaning

737 that it selected the maximum values from contiguous chunks of 13 adjacent outputs from the previous

738 layer. We applied dropout regularization (Srivastava et al., 2014) on the outputs of the max pooling

739 operation to prevent overfitting to the training set. We then flattened the outputs of the max pooling layer

740 into a single vector and passed them to a single output unit with a sigmoid activation function. We used

741 stochastic gradient descent (SGD) to minimize binary cross entropy loss (log loss) between the output of

742 this unit and the positive/negative class label to learn model parameters.

743 Each model was trained for 100 passes through the training set (or "epochs"). For the PV vs. PV-

744 and the PV vs. VIP tasks, we evaluated model performance and chose the best performing model based

745 on the value of the binary cross entropy loss on the validation set. For the PV vs. EXC task, we chose the

746 final model based on a combination of auROC and auPRC on the validation set. We ignored small

747 differences in validation auROC and auPRC $( \pm 0.02)$ while selecting the final PV vs. EXC model. Tuning

748 only the number of variable convolution layers $(0,1$, or 2$)$, and the dropout probability for the max 
pooling output $(0.2,0.4$, or 0.5$)$, we were able to achieve strong auROCs and auPRCs on the held out validation sets. Therefore, we did not attempt to vary learning rate for SGD (0.01), momentum (0.0), batch size (30), number of training epochs (100), number of filters in the first convolution layer (1000), number of filters in subsequent convolution layers (100), kernel sizes (8), max pooling size (13) and stride (13). A table of hyperparameter settings and associated performance metrics (loss value, auROC, auPRC) on training, validation, and test sets is provided in Supplemental Table 3. Fig. 2) were extracted from AAV plasmids with confirmed cell type-specific activity in vivo. The Gfap promoter sequence (Gfa2) was from hGFAP-GFP (Addgene plasmid \#40592;

http://n2t.net/addgene:40592; RRID:Addgene_40592). The CamkII promoter sequence was from pENN.AAV.CamKII0.4.eGFP.WPRE.rBG (Addgene plasmid \#105541; http://n2t.net/addgene:105541; RRID:Addgene_105541). The Dlx promoter sequence was from pAAV-mDlx-GFP-Fishell-1 (Addgene plasmid \#83900; http://n2t.net/addgene:83900; RRID:Addgene_83900)(Dimidschstein et al., 2016). sequences (Vormstein-Schneider et al., 2020) were scored through all cortical PV SVMs. To enable comparison between models, scores were normalized to standard deviations from 0 using the standard variation of the validation data set for each model. For each pair of models, the sequence scores were assessed for correlation with cor() function from the R Stats package

767 (https://www.rdocumentation.org/packages/stats/versions/3.6.2) with the Pearson method and visualized using the corrplot package in R (https://github.com/taiyun/corrplot) (Supplemental Fig. 4).

770 approaches for prioritizing enhancer candidates for cell type-specific AAV design include

$771 \log 2$ FoldDifference and conservation-based ranking. We show that machine learning models are more

772 predictive of success than these approaches by evaluating on the external PV enhancer AAV screen

773 (Vormstein-Schneider et al., 2020). The log2FoldDifference of ATAC-seq signal in different cell type

774 comparisons was evaluated from snATAC-seq data (Li et al., 2020). We added the exact genomic 
775 locations of each test sequence to the genomic peak set for assessment and applied the findDAR()

776 function with test.method = "exactTest" in SnapATAC version 1.0.0 (Fang et al., 2021). The

$777 \log 2$ FoldDifference was determined for i) the PV cluster relative to all PV - cells using cluster.neg =

778 "random", ii) the PV cluster relative to closely related cells using cluster.neg = "knn", iii) the PV cluster

779 relative to the pool of excitatory neuron clusters, iv) the PV cluster relative to the VIP cluster, and v) the

780 PV cluster relative to the SST cluster (Supplemental Fig. 5).

Euarchontoglires PhyloP scores were extracted for all bases within each PV enhancer candidate

782 using the UCSC Table Browser (phyloP60wayEuarchontoGlires track for the Grcm38/mm10 genome,

783 accessed March 2021) (Kuhn et al., 2013). Regions were mapped from mouse (mm10) to human (hg38)

784 using UCSC LiftOver, requiring a minimum ratio of bases that must remap of 0.1. All regions were

785 mappable between species. Finally, we assessed overlapping human PV neuron OCRs from motor cortex snATAC-seq (Bakken et al., 2020) using bedtools intersect (Quinlan and Hall, 2010). Any peak overlap

787 of at least $1 \mathrm{bp}$ was recorded as an overlapping peak.

DESeqDataSet object with variance stabilizing transformation in DESeq2 version 1.26.0 (Love et al.,

791 particular cell group comparisons by using the results contrasts. Correlations between

$792 \log 2$ FoldDifferences for PV cSNAIL vs. bulk tissue and log2FoldDifferences for SNAIL probes vs. bulk

793 tissue were assessed using the R function cor.test() with both "spearman" and "pearson" methods.

794 Genome browser tracks were visualized in the mm10 genome using IGV (Robinson et al., 2011) and track

795 heights were normalized between samples of the same experimental ATAC-seq method (cSNAIL,

796 SNAIL, bulk tissue, or single nucleus). Comparisons to snATAC-seq cluster markers (Fig. 3d,

797 Supplemental Fig. 8) represent the percentage of cSNAIL/SNAIL ATAC-seq OCRs enriched relative to

798 bulk (padj $<0.05 \& \log 2$ FoldDifference $>0.5$ ) that overlap snATAC-seq cluster markers. snATAC-seq

799 cluster markers were defined as enriched OCRs for that cluster relative to its k-nearest neighbors (padj <

$800 \quad 0.01 \& \log 2$ FoldDifference $>1)$ that were not enriched OCRs for any other cluster. The significance of 
801 the enrichments was assessed using the hypergeometric test with the phyper() function in R, setting

802 lower.tail = FALSE. Enrichments for cluster-specific OCRs were assessed using a background of all

803 snATAC-seq OCRs $(\mathrm{N}=415,813)$ and p-values were corrected for 84 tests with Bonferroni correction. Assessment of PV neuron OCRs in different brain regions. PV neuron cSNAIL ATAC-seq samples from cortex, striatum, and GPe tissue of healthy control mice from Lawler et al., 2020 (1 male, 1 female) were assessed for differential open chromatin using DESeq2 as described above. OCRs that were

807 preferentially open in one brain region relative to each of the other brain regions (padj $<0.01 \&$

$808 \log 2$ FoldDifference $>1$ ) were evaluated for sequence motif and pathway enrichments. Motif enrichments

809 for tissue-specific PV OCRs were identified using AME version 5.3.3 (Mc Leay and Bailey, 2010)

810 against a background of PV OCRs from all three tissues. Similarly, pathway enrichments using GREAT

811 version 4.0.4 (McLean et al., 2010) were carried out for tissue-specific PV OCRs relative to a background

812 of PV OCRs from all three tissues.

Model interpretation. We used GkmExplain (Shrikumar et al., 2019) to calculate actual and

814 hypothetical importance scores per base for each of 11 SVMs among 1,755 true positive PV-specific

815 OCR sequences that also scored PV-specific across all SVMs. First, sequences were one-hot encoded.

816 The importance scores were normalized based on the hypothetical importance scores of all possibilities

817 per base, so that a base position decreased in importance if there were other nucleotide possibilities that

818 produced similar scores. We identified sequence motifs with high contributions to PV scores for each

819 SVM separately using TF-MoDISco version 0.4.2.3 (Shrikumar et al., 2018) with options chosen to align

820 with final SVM parameters: sliding_window_size = 7, flank_size = 3, min_seqlets_per_task=3000,

821 trim_to_window_size $=7$, initial_flank_to_add $=3$, final_flank_to_add $=4$, kmer_len $=7$, num_gaps $=1$,

822 and num_mismatches $=1$. The resulting sequence patterns, representing motifs generated from seqlet

823 clusters, were trimmed to the 13 central bases and patterns with support from more than 100 seqlets were

824 used in downstream analysis. The position weight matrices (PWMs) of these patterns were associated

825 with known motifs in the Human and Mouse HOCOMOCO v11 FULL database using Tomtom (Gupta et

826 al., 2007) with the Pearson correlation coefficient motif comparison function (Supplemental Table 12). 
827 Motifs from all models were clustered based on PWM similarity using STAMP (Mahony and Benos,

828 2007); STAMP operations were performed after trimming motif edges with information content less than

8290.4 , using ungapped Smith-Waterman alignment, the iterative refinement multiple alignment strategy,

830 Pearson correlation coefficient comparison metrics, and UPGMA tree construction. Finally, individual

831 instances of motif sites were mapped in SC1 and SC2 sequences using FIMO with default parameters

832 (Grant et al., 2011).

834 Acknowledgements

835 This material is based upon work supported by NIH Grant DP1DA046585 and the National Science

836 Foundation Graduate Research Fellowship Grants DGE1252522 and DGE1745016.

\section{Competing Interests Statement}

839 AJL, ER, and ARP are inventors on US Patent Application 62/921,452, "Specific nuclear-anchored 840 independent labeling system”.

\section{Figure Legends}

843 Figure 1: Classification of neuron subtype-specific enhancer activity from sequence. a) Schematic

844 representation of the SNAIL workflow. b-e) Receiver operator characteristic and precision-recall

845 performance metrics for various cell type-specific enhancer sequence model strategies and data

846 modalities. The reported numbers are the areas under the curves for each model. f) Scatter plots for SVM

847 scores reported by equivalent population-derived models and single nucleus-derived models. *** p-value

848 of correlation $<0.001 . \mathrm{g}$ ) Top five sequence pattern contributors to PV prediction in linear, population-

849 derived SVMs. The best matching known motif is listed (full results in Supplemental Table 12).

851 Figure 2: Two sequences candidates selectively activate AAV expression in PV neurons. a) Genome

852 browser visualization of PV specific ATAC-seq signal at sequence candidates SC1 and SC2. * cSNAIL 

SVM scores among 1,755 true PV-specific enhancer sequence candidates that scored positively across all models. Linear population-derived models are denoted with “pop”, nonlinear population-derived models are denoted with "pop, rbf", and linear single nucleus-derived models are denoted with "sn". c) Example images of AAV Sun1GFP expression against parvalbumin (Pvalb) antibody staining. d,e) Quantification of AAV Sun1GFP or Cre reporter overlap with Pvalb+ cells. Bar heights represent the mean among images and the error of the mean is shown. $\mathrm{N}$ cells = 1,322 (SC1), 2,570 (SC2), 1,340 (Cre), 2,013 (Ef1a), and 504 (N.C.). N.C = negative control.

Figure 3: Cortical SC1 and SC2 SNAIL-isolated nuclei recapitulate PV GABAergic interneuron ATAC-seq signatures. a) PCA of ATAC-seq counts across samples. b) Genome browser visualization of ATAC-seq signal at the Pvalb gene locus. Tracks represent the pooled sample p-value signal. Each track of similar data type is normalized to the same scale: SNAIL data range 0 - 335, * cSNAIL data range 0 93, †INTACT data range 0 - 200, $\$$ snATAC-seq data range 0 - 2. c) Scatter plots of ATAC-seq $\log 2$ fold difference relative to bulk tissue ATAC-seq, comparing PV cSNAIL to other AAVs. The density of overlapping points is shown by the plot color. d) snATAC-seq nuclei clusters as visualized by t-SNE. The dendrograms show hierarchical clustering of Euclidean sample distances by Ward's minimum variance method D2. The heatmap shows the percentage of population OCRs enriched relative to bulk that are also

871 cluster-specific marker OCRs. * Hypergeometric enrichment $\mathrm{p}<0.01$.

873 Figure 4: SC1 and SC2 generalize to PV neurons in the striatum and GPe. a) Numbers of differential

874 OCRs between PV neuron populations in three brain regions (DESeq2 padj $<0.01 \& \mid \log 2$ FoldDifference $\mid$

875 > 1). Brain region-specific OCRs are those that were significantly enriched in that tissue relative to each

876 of the other two tissues. OCRs shared between two brain regions on the venn diagram are those that were

877 significantly enriched in each of those tissues relative to the excluded tissue. The shared center of the

878 venn diagram shows all remaining OCRs that have ambiguous or no tissue preference. b) Examples of 
enriched motifs in brain region-specific PV open chromatin relative to all PV open chromatin. c,f)

Distributions of validation data SVM scores and SC1 and SC2 scores within striatum and GPe PV vs PV-

models. d,g) PCA visualization of ATAC-seq counts in each sample. e,h) Pearson correlation coefficients

when comparing the $\log 2$ fold difference of cSNAIL PV ATAC-seq relative to bulk tissue ATAC-seq and

the $\log 2$ fold difference of SNAIL ATAC-seq relative to bulk tissue ATAC-seq. Error bars show the 95\%

confidence intervals.

Figure 5: Motif interpretation of PV neuron-specific OCR activity. a) Motifs with high contributions

to PV scores in each SVM, clustered by sequence similarity. The bubble color at each node shows the

model that motif was discovered in and the size of the bubble shows the number of seqlets supporting that

motif. Clusters are labeled by the clade majority best match for known transcription factor binding motifs.

The full list of matches can be found in Supplemental Table 12. b,c) Normalized importance of each base

in SC1 (b) and SC2 (c) sequences for their PV-specific scores in each SVM. Locations with sequence

matches for identified motifs in each SVM (from panel a) are shown at the bottom.

\section{References}

Amemiya HM, Kundaje A, Boyle AP. 2019. The ENCODE Blacklist: Identification of Problematic Regions of the Genome. Sci Rep 9:9354.

Bakken TE, Jorstad NL, Hu Q, Lake BB, Tian W, Kalmbach BE, Crow M, Hodge RD, Krienen FM, Sorensen SA, Eggermont J, Yao Z, Aevermann BD, Aldridge AI, Bartlett A, Bertagnolli D, Casper T, Castanon RG, Crichton K, Daigle TL, Dalley R, Dee N, Dembrow N, Diep D, Ding S-L, Dong W, Fang R, Fischer S, Goldman M, Goldy J, Graybuck LT, Herb BR, Hou X, Kancherla J, Kroll M, Lathia K, van Lew B, Li YE, Liu CS, Liu H, Lucero JD, Mahurkar A, McMillen D, Miller JA, Moussa M, Nery JR, Nicovich PR, Orvis J, Osteen JK, Owen S, Palmer CR, Pham T, Plongthongkum N, Poirion O, Reed NM, Rimorin C, Rivkin A, Romanow WJ, Sedeño-Cortés AE, Siletti K, Somasundaram S, Sulc J, Tieu M, Torkelson A, Tung H, Wang X, Xie F, Yanny AM, Zhang R, Ament SA, Margarita Behrens M, Bravo HC, Chun J, Dobin A, Gillis J, Hertzano R, Hof PR, Höllt T, Horwitz GD, Dirk Keene C, Kharchenko PV, Ko AL, Lelieveldt BP, Luo C, Mukamel EA, Preissl S, Regev A, Ren B, Scheuermann RH, Smith K, Spain WJ, White OR, Koch C, Hawrylycz M, Tasic B, Macosko EZ, McCarroll SA, Ting JT, Zeng H, Zhang K, Feng G, Ecker JR, Linnarsson S, Lein ES. 2020. Evolution of cellular diversity in primary motor cortex of human, marmoset monkey, and mouse. bioRxiv. doi:10.1101/2020.03.31.016972

Buenrostro JD, Giresi PG, Zaba LC, Chang HY, Greenleaf WJ. 2013. Transposition of native chromatin for fast and sensitive epigenomic profiling of open chromatin, DNA-binding proteins and nucleosome position. Nat Methods 10:1213-1218.

914 Buenrostro JD, Wu B, Chang HY, Greenleaf WJ. 2015a. ATAC-seq: A Method for Assaying Chromatin 
Accessibility Genome-Wide. Curr Protoc Mol Biol 109:21.29.1-21.29.9.

Buenrostro JD, Wu B, Litzenburger UM, Ruff D, Gonzales ML, Snyder MP, Chang HY, Greenleaf WJ. 2015b. Single-cell chromatin accessibility reveals principles of regulatory variation. Nature 523:486-490.

Chan KY, Jang MJ, Yoo BB, Greenbaum A, Ravi N, Wu W-L, Sánchez-Guardado L, Lois C, Mazmanian SK, Deverman BE, Gradinaru V. 2017. Engineered AAVs for efficient noninvasive gene delivery to the central and peripheral nervous systems. Nat Neurosci 20:1172-1179.

Chen L, Fish AE, Capra JA. 2018. Prediction of gene regulatory enhancers across species reveals evolutionarily conserved sequence properties. PLoS Comput Biol 14:e1006484.

Cochran K, Srivastava D, Shrikumar A, Balsubramani A, Kundaje A, Mahony S. 2021. Domain adaptive neural networks improve cross-species prediction of transcription factor binding. bioRxiv. doi:10.1101/2021.02.13.431115

Cun YL, Jackel LD, Boser B, Denker JS, Graf HP, Guyon I, Henderson D, Howard RE, Hubbard W. 1989. Handwritten digit recognition: applications of neural network chips and automatic learning. IEEE Communications Magazine 27:41-46.

Cusanovich DA, Daza R, Adey A, Pliner HA, Christiansen L, Gunderson KL, Steemers FJ, Trapnell C, Shendure J. 2015. Multiplex single cell profiling of chromatin accessibility by combinatorial cellular indexing. Science 348:910-914.

Deal RB, Henikoff S. 2010. A simple method for gene expression and chromatin profiling of individual cell types within a tissue. Dev Cell 18:1030-1040.

Deverman BE, Pravdo PL, Simpson BP, Kumar SR, Chan KY, Banerjee A, Wu W-L, Yang B, Huber N, Pasca SP, Gradinaru V. 2016. Cre-dependent selection yields AAV variants for widespread gene transfer to the adult brain. Nat Biotechnol 34:204-209.

Dimidschstein J, Chen Q, Tremblay R, Rogers SL, Saldi G-A, Guo L, Xu Q, Liu R, Lu C, Chu J, Grimley JS, Krostag A-R, Kaykas A, Avery MC, Rashid MS, Baek M, Jacob AL, Smith GB, Wilson DE, Kosche G, Kruglikov I, Rusielewicz T, Kotak VC, Mowery TM, Anderson SA, Callaway EM, Dasen JS, Fitzpatrick D, Fossati V, Long MA, Noggle S, Reynolds JH, Sanes DH, Rudy B, Feng G, Fishell G. 2016. A viral strategy for targeting and manipulating interneurons across vertebrate species. Nat Neurosci 19:1743-1749.

Donato F, Chowdhury A, Lahr M, Caroni P. 2015. Early- and late-born parvalbumin basket cell subpopulations exhibiting distinct regulation and roles in learning. Neuron 85:770-786.

Fang R, Preissl S, Li Y, Hou X, Lucero J, Wang X, Motamedi A, Shiau AK, Zhou X, Xie F, Mukamel EA, Zhang K, Zhang Y, Behrens MM, Ecker JR, Ren B. 2021. Comprehensive analysis of single cell ATAC-seq data with SnapATAC. Nat Commun 12:1337.

Ghandi M, Lee D, Mohammad-Noori M, Beer MA. 2014. Enhanced regulatory sequence prediction using gapped k-mer features. PLoS Comput Biol 10:e1003711.

Grant CE, Bailey TL, Noble WS. 2011. FIMO: scanning for occurrences of a given motif. Bioinformatics 27:1017-1018.

Graybuck LT, Daigle TL, Sedeño-Cortés AE, Walker M, Kalmbach B, Lenz GH, Morin E, Nguyen TN, Garren E, Bendrick JL, Kim TK, Zhou T, Mortrud M, Yao S, Siverts LA, Larsen R, Gore BB, Szelenyi ER, Trader C, Balaram P, van Velthoven CTJ, Chiang M, Mich JK, Dee N, Goldy J, Cetin AH, Smith K, Way SW, Esposito L, Yao Z, Gradinaru V, Sunkin SM, Lein E, Levi BP, Ting JT, Zeng H, Tasic B. 2021. Enhancer viruses for combinatorial cell-subclass-specific labeling. Neuron. doi:10.1016/j.neuron.2021.03.011

Gupta S, Stamatoyannopoulos JA, Bailey TL, Noble WS. 2007. Quantifying similarity between motifs. Genome Biol 8:R24.

Hernández VM, Hegeman DJ, Cui Q, Kelver DA, Fiske MP, Glajch KE, Pitt JE, Huang TY, Justice NJ, Savio Chan C. 2015. Parvalbumin+ Neurons and Npas1+ Neurons Are Distinct Neuron Classes in the Mouse External Globus Pallidus. J Neurosci 35:11830-11847.

Hodge RD, Bakken TE, Miller JA, Smith KA, Barkan ER, Graybuck LT, Close JL, Long B, Johansen N, Penn O, Yao Z, Eggermont J, Höllt T, Levi BP, Shehata SI, Aevermann B, Beller A, Bertagnolli D, 
1001

1002

1003

1004

1005

1006

1007

1008

1009

1010

1011

1012

1013

1014

1015

1016

Brouner K, Casper T, Cobbs C, Dalley R, Dee N, Ding S-L, Ellenbogen RG, Fong O, Garren E, Goldy J, Gwinn RP, Hirschstein D, Keene CD, Keshk M, Ko AL, Lathia K, Mahfouz A, Maltzer Z, McGraw M, Nguyen TN, Nyhus J, Ojemann JG, Oldre A, Parry S, Reynolds S, Rimorin C, Shapovalova NV, Somasundaram S, Szafer A, Thomsen ER, Tieu M, Quon G, Scheuermann RH, Yuste R, Sunkin SM, Lelieveldt B, Feng D, Ng L, Bernard A, Hawrylycz M, Phillips JW, Tasic B, Zeng H, Jones AR, Koch C, Lein ES. 2019. Conserved cell types with divergent features in human versus mouse cortex. Nature 573:61-68.

Hoffman MM, Ernst J, Wilder SP, Kundaje A, Harris RS, Libbrecht M, Giardine B, Ellenbogen PM, Bilmes JA, Birney E, Hardison RC, Dunham I, Kellis M, Noble WS. 2013. Integrative annotation of chromatin elements from ENCODE data. Nucleic Acids Res 41:827-841.

Hrvatin S, Tzeng CP, Nagy MA, Stroud H, Koutsioumpa C, Wilcox OF, Assad EG, Green J, Harvey CD, Griffith EC, Greenberg ME. 2019. A scalable platform for the development of cell-type-specific viral drivers. eLife 2019:e48089.

Jindal GA, Farley EK. 2021. Enhancer grammar in development, evolution, and disease: dependencies and interplay. Dev Cell 56:575-587.

Jinno S, Kosaka T. 2004. Parvalbumin is expressed in glutamatergic and GABAergic corticostriatal pathway in mice. J Comp Neurol 477:188-201.

Kaplow IM, Wirthlin ME, Lawler AJ, Brown AR, Kleyman M, Pfenning AR. 2020. Predicting lineagespecific differences in open chromatin across dozens of mammalian genomes. bioRxiv. doi:10.1101/2020.12.04.410795

Kelley DR. 2020. Cross-species regulatory sequence activity prediction. PLoS Comput Biol 16:e1008050. Kelley DR, Snoek J, Rinn JL. 2016. Basset: learning the regulatory code of the accessible genome with deep convolutional neural networks. Genome Res 26:990-999.

Kellis M, Wold B, Snyder MP, Bernstein BE, Kundaje A, Marinov GK, Ward LD, Birney E, Crawford GE, Dekker J, Dunham I, Elnitski LL, Farnham PJ, Feingold EA, Gerstein M, Giddings MC, Gilbert DM, Gingeras TR, Green ED, Guigo R, Hubbard T, Kent J, Lieb JD, Myers RM, Pazin MJ, Ren B, Stamatoyannopoulos JA, Weng Z, White KP, Hardison RC. 2014. Defining functional DNA elements in the human genome. Proc Natl Acad Sci U S A 111:6131-6138.

Kepecs A, Fishell G. 2014. Interneuron cell types are fit to function. Nature 505:318-326.

Khan A, Zhang X. 2016. dbSUPER: a database of super-enhancers in mouse and human genome. Nucleic Acids Res 44:D164-71.

Korsunsky I, Millard N, Fan J, Slowikowski K, Zhang F, Wei K, Baglaenko Y, Brenner M, Loh P-R, Raychaudhuri S. 2019. Fast, sensitive and accurate integration of single-cell data with Harmony. Nat Methods 16:1289-1296.

Kuhn RM, Haussler D, Kent WJ. 2013. The UCSC genome browser and associated tools. Brief Bioinform 14:144-161.

Lake BB, Ai R, Kaeser GE, Salathia NS, Yung YC, Liu R, Wildberg A, Gao D, Fung H-L, Chen S, Vijayaraghavan R, Wong J, Chen A, Sheng X, Kaper F, Shen R, Ronaghi M, Fan J-B, Wang W, Chun J, Zhang K. 2016. Neuronal subtypes and diversity revealed by single-nucleus RNA sequencing of the human brain. Science 352:1586-1590.

Lawler AJ, Brown AR, Bouchard RS, Toong N, Kim Y, Velraj N, Fox G, Kleyman M, Kang B, Gittis AH, Pfenning AR. 2020. Cell Type-Specific Oxidative Stress Genomic Signatures in the Globus Pallidus of Dopamine-Depleted Mice. J Neurosci 40:9772-9783.

Lee D. 2016. LS-GKM: a new gkm-SVM for large-scale datasets. Bioinformatics 32:2196-2198.

Lee JH, Durand R, Gradinaru V, Zhang F, Goshen I, Kim D-S, Fenno LE, Ramakrishnan C, Deisseroth K. 2010. Global and local fMRI signals driven by neurons defined optogenetically by type and wiring. Nature 465:788-792.

Liao Y, Smyth GK, Shi W. 2019. The R package Rsubread is easier, faster, cheaper and better for alignment and quantification of RNA sequencing reads. Nucleic Acids Res 47:e47.

Liao Y, Smyth GK, Shi W. 2014. featureCounts: an efficient general purpose program for assigning sequence reads to genomic features. Bioinformatics 30:923-930. 
1051

1052

1053

1054

1055

1056

1057

1058

1059

1060

1061

1062

1063

1064

1065

1066

1067
Lim L, Mi D, Llorca A, Marín O. 2018. Development and Functional Diversification of Cortical Interneurons. Neuron 100:294-313.

Lin J, Handschin C, Spiegelman BM. 2005. Metabolic control through the PGC-1 family of transcription coactivators. Cell Metab 1:361-370.

Liodis P, Denaxa M, Grigoriou M, Akufo-Addo C, Yanagawa Y, Pachnis V. 2007. Lhx6 activity is required for the normal migration and specification of cortical interneuron subtypes. $J$ Neurosci 27:3078-3089.

Li Q, Brown JB, Huang H, Bickel PJ. 2011. Measuring reproducibility of high-throughput experiments. aoas 5:1752-1779.

Li YE, Preissl S, Hou X, Zhang Z, Zhang K, Fang R, Qiu Y, Poirion O, Li B, Liu H, Wang X, Han JY, Lucero J, Yan Y, Kuan S, Gorkin D, Nunn M, Mukamel EA, Margarita Behrens M, Ecker J, Ren B. 2020. An Atlas of Gene Regulatory Elements in Adult Mouse Cerebrum. bioRxiv. doi:10.1101/2020.05.10.087585

Love MI, Huber W, Anders S. 2014. Moderated estimation of fold change and dispersion for RNA-seq data with DESeq2. Genome Biol 15:550-550.

Lucas EK, Dougherty SE, McMeekin LJ, Reid CS, Dobrunz LE, West AB, Hablitz JJ, Cowell RM. 2014. PGC-1 $\alpha$ provides a transcriptional framework for synchronous neurotransmitter release from parvalbumin-positive interneurons. J Neurosci 34:14375-14387.

Lucas EK, Markwardt SJ, Gupta S, Meador-Woodruff JH, Lin JD, Overstreet-Wadiche L, Cowell RM. 2010. Parvalbumin deficiency and GABAergic dysfunction in mice lacking PGC-1alpha. J Neurosci 30:7227-7235.

Madisen L, Zwingman TA, Sunkin SM, Oh SW, Zariwala HA, Gu H, Ng LL, Palmiter RD, Hawrylycz MJ, Jones AR, Lein ES, Zeng H. 2010. A robust and high-throughput Cre reporting and characterization system for the whole mouse brain. Nat Neurosci 13:133-140.

Mahony S, Benos PV. 2007. STAMP: a web tool for exploring DNA-binding motif similarities. Nucleic Acids Res 35:W253-8.

Mayer C, Hafemeister C, Bandler RC, Machold R, Batista Brito R, Jaglin X, Allaway K, Butler A, Fishell G, Satija R. 2018. Developmental diversification of cortical inhibitory interneurons. Nature 555:457-462.

McLean CY, Bristor D, Hiller M, Clarke SL, Schaar BT, Lowe CB, Wenger AM, Bejerano G. 2010. GREAT improves functional interpretation of cis-regulatory regions. Nat Biotechnol 28:495-501.

Mc Leay RC, Bailey TL. 2010. and an evaluation on ChIP data. McLeay and Bailey BMC Bioinformatics 11:165.

Mich JK, Graybuck LT, Hess EE, Mahoney JT, Kojima Y, Ding Y, Somasundaram S, Miller JA, Kalmbach BE, Radaelli C, Gore BB, Weed N, Omstead V, Bishaw Y, Shapovalova NV, Martinez RA, Fong O, Yao S, Mortrud M, Chong P, Loftus L, Bertagnolli D, Goldy J, Casper T, Dee N, Opitz-Araya X, Cetin A, Smith KA, Gwinn RP, Cobbs C, Ko AL, Ojemann JG, Keene CD, Silbergeld DL, Sunkin SM, Gradinaru V, Horwitz GD, Zeng H, Tasic B, Lein ES, Ting JT, Levi BP. 2021. Functional enhancer elements drive subclass-selective expression from mouse to primate neocortex. Cell Rep 34:108754.

Minnoye L, Taskiran II, Mauduit D, Fazio M, Van Aerschot L, Hulselmans G, Christiaens V, Makhzami S, Seltenhammer M, Karras P, Primot A, Cadieu E, van Rooijen E, Marine J-C, Egidy G, Ghanem GE, Zon L, Wouters J, Aerts S. 2020. Cross-species analysis of enhancer logic using deep learning. Genome Res 30:1815-1834.

Mitchell AC, Javidfar B, Pothula V, Ibi D, Shen EY, Peter CJ, Bicks LK, Fehr T, Jiang Y, Brennand KJ, Neve RL, Gonzalez-Maeso J, Akbarian S. 2018. MEF2C transcription factor is associated with the genetic and epigenetic risk architecture of schizophrenia and improves cognition in mice. Mol Psychiatry 23:123-132.

Mo A, Mukamel EA, Davis FP, Luo C, Henry GL, Picard S, Urich MA, Nery JR, Sejnowski TJ, Lister R, Eddy SR, Ecker JR, Nathans J. 2015. Epigenomic Signatures of Neuronal Diversity in the Mammalian Brain. Neuron 86:1369-1384. 
1068

1069

1070

1071

1072

1073

1074

1075

1076

1077

1078

1079

1080

1081

1082

1083

1084

1085

1086

1087

1088

1089

1090

1091

1092

1093

1094

1095

1096

1097

1098

1099

1100

1101

1102

1103

1104

1105

1106

1107

1108

1109

1110

1111

1112

1113

1114

1115

1116

1117

1118

Nair RR, Blankvoort S, Lagartos MJ, Kentros C. 2020. Enhancer-Driven Gene Expression (EDGE) Enables the Generation of Viral Vectors Specific to Neuronal Subtypes. iScience 23:100888.

Nathanson JL, Jappelli R, Scheeff ED, Manning G, Obata K, Brenner S, Callaway EM. 2009. Short Promoters in Viral Vectors Drive Selective Expression in Mammalian Inhibitory Neurons, but do not Restrict Activity to Specific Inhibitory Cell-Types. Front Neural Circuits 3:19.

Pai EL-L, Chen J, Fazel Darbandi S, Cho FS, Chen J, Lindtner S, Chu JS, Paz JT, Vogt D, Paredes MF, Rubenstein JL. 2020. Maf and Mafb control mouse pallial interneuron fate and maturation through neuropsychiatric disease gene regulation. eLife 2020:e54903.

Paul A, Crow M, Raudales R, He M, Gillis J, Huang ZJ. 2017. Transcriptional Architecture of Synaptic Communication Delineates GABAergic Neuron Identity. Cell 171:522-539.e20.

Preissl S, Fang R, Huang H, Zhao Y, Raviram R, Gorkin DU, Zhang Y, Sos BC, Afzal V, Dickel DE, Kuan S, Visel A, Pennacchio LA, Zhang K, Ren B. 2018. Single-nucleus analysis of accessible chromatin in developing mouse forebrain reveals cell-type-specific transcriptional regulation. Nat Neurosci 21:432-439.

Quang D, Xie X. 2016. DanQ: a hybrid convolutional and recurrent deep neural network for quantifying the function of DNA sequences. Nucleic Acids Res 44:e107-e107.

Quinlan AR, Hall IM. 2010. BEDTools: a flexible suite of utilities for comparing genomic features. Bioinformatics 26:841-842.

Roadmap Epigenomics Consortium, Kundaje A, Meuleman W, Ernst J, Bilenky M, Yen A, HeraviMoussavi A, Kheradpour P, Zhang Z, Wang J, Ziller MJ, Amin V, Whitaker JW, Schultz MD, Ward LD, Sarkar A, Quon G, Sandstrom RS, Eaton ML, Wu Y-C, Pfenning AR, Wang X, Claussnitzer M, Liu Y, Coarfa C, Harris RA, Shoresh N, Epstein CB, Gjoneska E, Leung D, Xie W, Hawkins RD, Lister R, Hong C, Gascard P, Mungall AJ, Moore R, Chuah E, Tam A, Canfield TK, Hansen RS, Kaul R, Sabo PJ, Bansal MS, Carles A, Dixon JR, Farh K-H, Feizi S, Karlic R, Kim A-R, Kulkarni A, Li D, Lowdon R, Elliott G, Mercer TR, Neph SJ, Onuchic V, Polak P, Rajagopal N, Ray P, Sallari RC, Siebenthall KT, Sinnott-Armstrong NA, Stevens M, Thurman RE, Wu J, Zhang B, Zhou X, Beaudet AE, Boyer LA, De Jager PL, Farnham PJ, Fisher SJ, Haussler D, Jones SJM, Li W, Marra MA, McManus MT, Sunyaev S, Thomson JA, Tlsty TD, Tsai L-H, Wang W, Waterland RA, Zhang MQ, Chadwick LH, Bernstein BE, Costello JF, Ecker JR, Hirst M, Meissner A, Milosavljevic A, Ren B, Stamatoyannopoulos JA, Wang T, Kellis M. 2015. Integrative analysis of 111 reference human epigenomes. Nature 518:317-330.

Robinson JT, Thorvaldsdóttir H, Winckler W, Guttman M, Lander ES, Getz G, Mesirov JP. 2011. Integrative genomics viewer. Nat Biotechnol 29:24-26.

Roccaro-Waldmeyer DM, Girard F, Milani D, Vannoni E, Prétôt L, Wolfer DP, Celio MR. 2018. Eliminating the VGlut2-dependent glutamatergic transmission of parvalbumin-expressing neurons leads to deficits in locomotion and vocalization, decreased pain sensitivity, and increased dominance. Front Behav Neurosci 12:146.

Sakata K, Woo NH, Martinowich K, Greene JS, Schloesser RJ, Shen L, Lu B. 2009. Critical role of promoter IV-driven BDNF transcription in GABAergic transmission and synaptic plasticity in the prefrontal cortex. Proc Natl Acad Sci U S A 106:5942-5947.

Saunders A, Huang KW, Sabatini BL. 2016. Globus Pallidus Externus Neurons Expressing parvalbumin Interconnect the Subthalamic Nucleus and Striatal Interneurons. PLoS One 11:e0149798.

Saunders A, Macosko EZ, Wysoker A, Goldman M, Krienen FM, de Rivera H, Bien E, Baum M, Bortolin L, Wang S, Goeva A, Nemesh J, Kamitaki N, Brumbaugh S, Kulp D, McCarroll SA. 2018. Molecular Diversity and Specializations among the Cells of the Adult Mouse Brain. Cell 174:10151030.e16.

Schindelin J, Arganda-Carreras I, Frise E, Kaynig V, Longair M, Pietzsch T, Preibisch S, Rueden C, Saalfeld S, Schmid B, Tinevez J-Y, White DJ, Hartenstein V, Eliceiri K, Tomancak P, Cardona A. 2012. Fiji: an open-source platform for biological-image analysis. Nat Methods 9:676-682.

Shima Y, Sugino K, Hempel CM, Shima M, Taneja P, Bullis JB, Mehta S, Lois C, Nelson SB. 2016. A Mammalian enhancer trap resource for discovering and manipulating neuronal cell types. Elife 
Shrikumar A, Prakash E, Kundaje A. 2019. GkmExplain: fast and accurate interpretation of nonlinear gapped k-mer SVMs. Bioinformatics 35:i173-i182.

Shrikumar A, Tian K, Shcherbina A. 2018. Technical Note on Transcription Factor Motif Discovery from Importance Scores (TF-MoDISco) version 0.4.2.2. arXiv.

Srivastava N, Hinton G, Krizhevsky A, Sutskever I, Salakhutdinov R. 2014. Dropout: a simple way to prevent neural networks from overfitting. JMLR 15:1929-1958.

Tanahira C, Higo S, Watanabe K, Tomioka R, Ebihara S, Kaneko T, Tamamaki N. 2009. Parvalbumin neurons in the forebrain as revealed by parvalbumin-Cre transgenic mice. Neurosci Res 63:213-223.

Taniguchi H, He M, Wu P, Kim S, Paik R, Sugino K, Kvitsiani D, Fu Y, Lu J, Lin Y, Miyoshi G, Shima Y, Fishell G, Nelson SB, Huang ZJ. 2011. A resource of Cre driver lines for genetic targeting of GABAergic neurons in cerebral cortex. Neuron 71:995-1013.

Taniguchi K, Anderson AE, Sutherland AE, Wotton D. 2012. Loss of Tgif function causes holoprosencephaly by disrupting the SHH signaling pathway. PLoS Genet 8:e1002524.

Tasic B, Yao Z, Graybuck LT, Smith KA, Nguyen TN, Bertagnolli D, Goldy J, Garren E, Economo MN, Viswanathan S, Penn O, Bakken T, Menon V, Miller J, Fong O, Hirokawa KE, Lathia K, Rimorin C, Tieu M, Larsen R, Casper T, Barkan E, Kroll M, Parry S, Shapovalova NV, Hirschstein D, Pendergraft J, Sullivan HA, Kim TK, Szafer A, Dee N, Groblewski P, Wickersham I, Cetin A, Harris JA, Levi BP, Sunkin SM, Madisen L, Daigle TL, Looger L, Bernard A, Phillips J, Lein E, Hawrylycz M, Svoboda K, Jones AR, Koch C, Zeng H. 2018. Shared and distinct transcriptomic cell types across neocortical areas. Nature 563:72-78.

The Theano Development Team, Al-Rfou R, Alain G, Almahairi A, Angermueller C, Bahdanau D, Ballas N, Bastien F, Bayer J, Belikov A, Belopolsky A, Bengio Y, Bergeron A, Bergstra J, Bisson V, Snyder JB, Bouchard N, Boulanger-Lewandowski N, Bouthillier X, de Brébisson A, Breuleux O, Carrier P-L, Cho K, Chorowski J, Christiano P, Cooijmans T, Côté M-A, Côté M, Courville A, Dauphin YN, Delalleau O, Demouth J, Desjardins G, Dieleman S, Dinh L, Ducoffe M, Dumoulin V, Kahou SE, Erhan D, Fan Z, Firat O, Germain M, Glorot X, Goodfellow I, Graham M, Gulcehre C, Hamel P, Harlouchet I, Heng J-P, Hidasi B, Honari S, Jain A, Jean S, Jia K, Korobov M, Kulkarni V, Lamb A, Lamblin P, Larsen E, Laurent C, Lee S, Lefrancois S, Lemieux S, Léonard N, Lin Z, Livezey JA, Lorenz C, Lowin J, Ma Q, Manzagol P-A, Mastropietro O, McGibbon RT, Memisevic R, van Merriënboer B, Michalski V, Mirza M, Orlandi A, Pal C, Pascanu R, Pezeshki M, Raffel C, Renshaw D, Rocklin M, Romero A, Roth M, Sadowski P, Salvatier J, Savard F, Schlüter J, Schulman J, Schwartz G, Serban IV, Serdyuk D, Shabanian S, Simon É, Spieckermann S, Ramana Subramanyam S, Sygnowski J, Tanguay J, van Tulder G, Turian J, Urban S, Vincent P, Visin F, de Vries H, Warde-Farley D, Webb DJ, Willson M, Xu K, Xue L, Yao L, Zhang S, Zhang Y. 2016. Theano: A Python framework for fast computation of mathematical expressions. arXiv.

Vogt D, Hunt RF, Mandal S, Sandberg M, Silberberg SN, Nagasawa T, Yang Z, Baraban SC, Rubenstein JLR. 2014. Lhx6 directly regulates Arx and CXCR7 to determine cortical interneuron fate and laminar position. Neuron 82:350-364.

Vormstein-Schneider D, Lin JD, Pelkey KA, Chittajallu R, Guo B, Arias-Garcia MA, Allaway K, Sakopoulos S, Schneider G, Stevenson O, Vergara J, Sharma J, Zhang Q, Franken TP, Smith J, Ibrahim LA, M Astro KJ, Sabri E, Huang S, Favuzzi E, Burbridge T, Xu Q, Guo L, Vogel I, Sanchez V, Saldi GA, Gorissen BL, Yuan X, Zaghloul KA, Devinsky O, Sabatini BL, Batista-Brito R, Reynolds J, Feng G, Fu Z, McBain CJ, Fishell G, Dimidschstein J. 2020. Viral manipulation of functionally distinct interneurons in mice, non-human primates and humans. Nat Neurosci 23:16291636.

Wolock SL, Lopez R, Klein AM. 2019. Scrublet: Computational Identification of Cell Doublets in Single-Cell Transcriptomic Data. Cell Syst 8:281-291.e9.

Zeisel A, Muñoz-Manchado AB, Codeluppi S, Lönnerberg P, La Manno G, Juréus A, Marques S, Munguba H, He L, Betsholtz C, Rolny C, Castelo-Branco G, Hjerling-Leffler J, Linnarsson S. 2015. Brain structure. Cell types in the mouse cortex and hippocampus revealed by single-cell RNA-seq. 
Zhang Y, Liu T, Meyer CA, Eeckhoute J, Johnson DS, Bernstein BE, Nusbaum C, Myers RM, Brown M, Li W, Liu XS. 2008. Model-based analysis of ChIP-Seq (MACS). Genome Biol 9:R137. pathways for development of telencephalic interneuron subtypes revealed through analysis of Lhx6 mutants. J Comp Neurol 510:79-99.

Zhou J, Troyanskaya OG. 2015. Predicting effects of noncoding variants with deep learning-based sequence model. Nat Methods 12:931-934. 


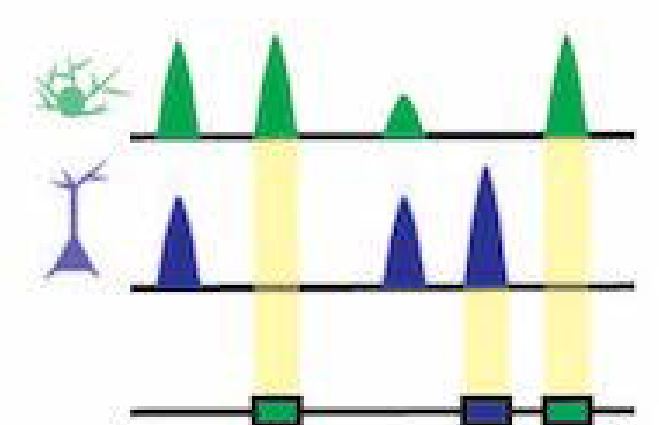

Sequences underlying cell type-specific open chromatin

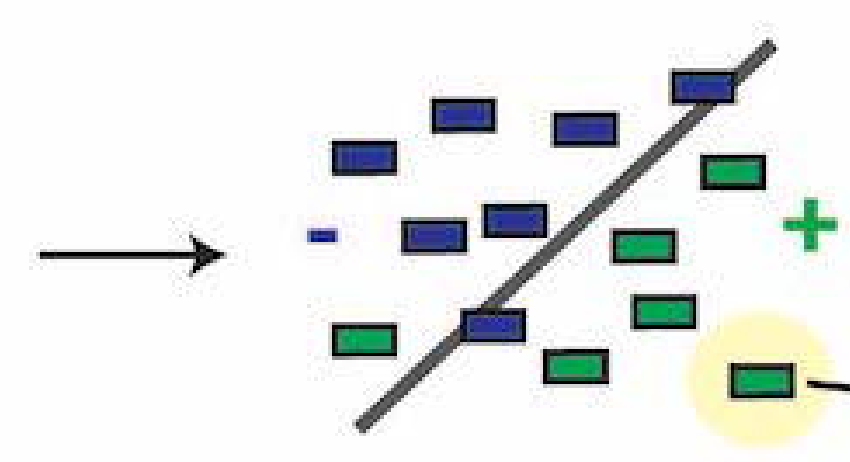

Sequence prioritization with machine learning classifiers

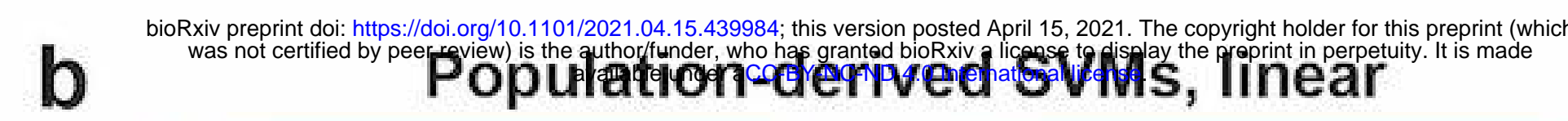

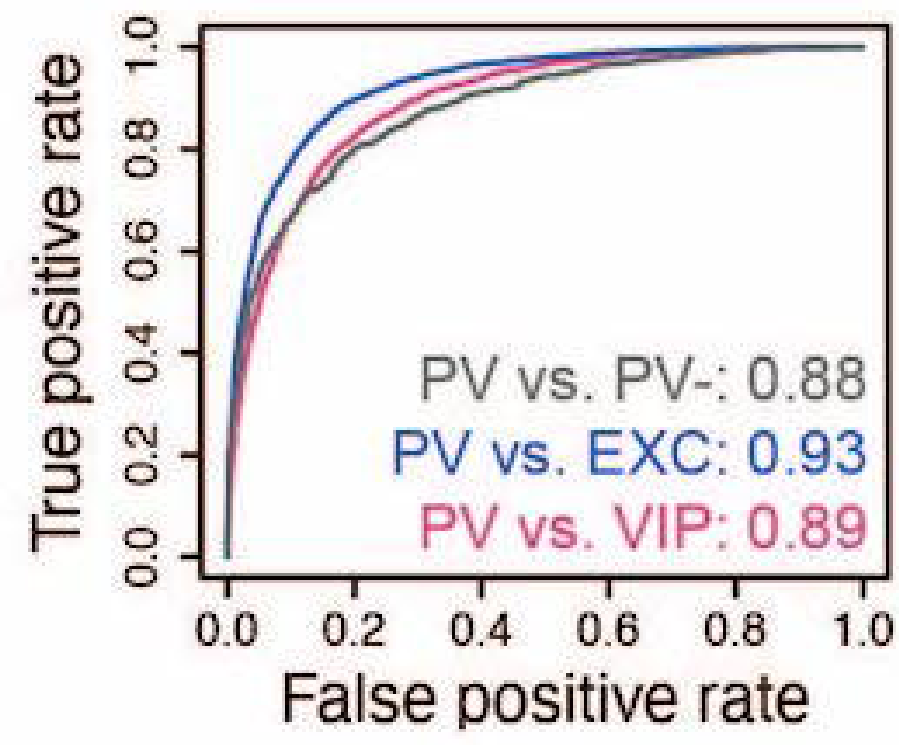

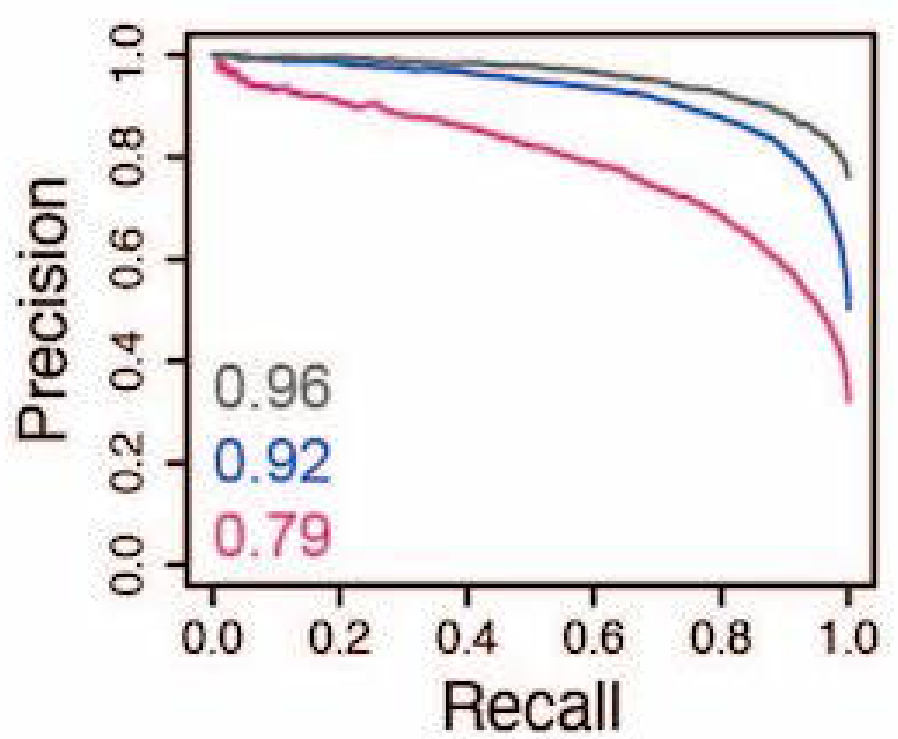

d

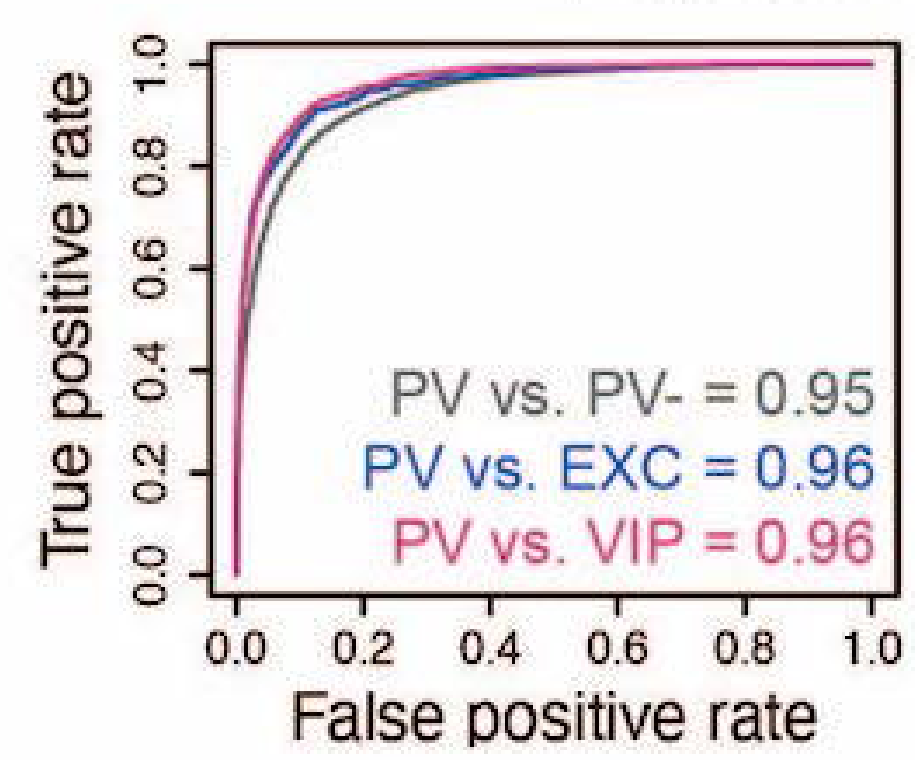

Population-derived CNNs

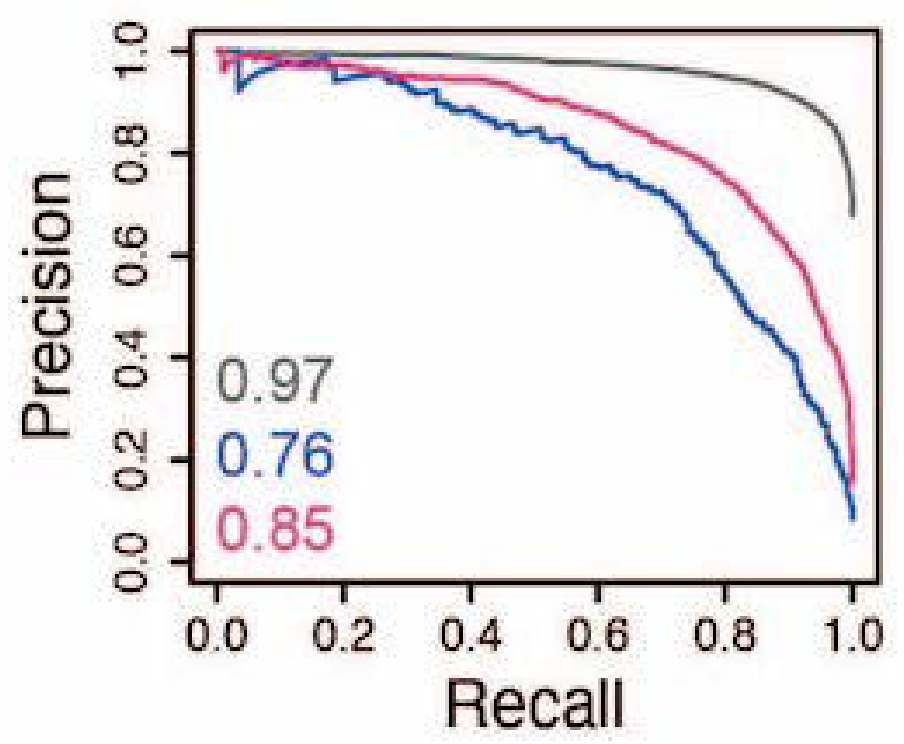

Package top candidates into AAV
Cell type-specific AAV expression

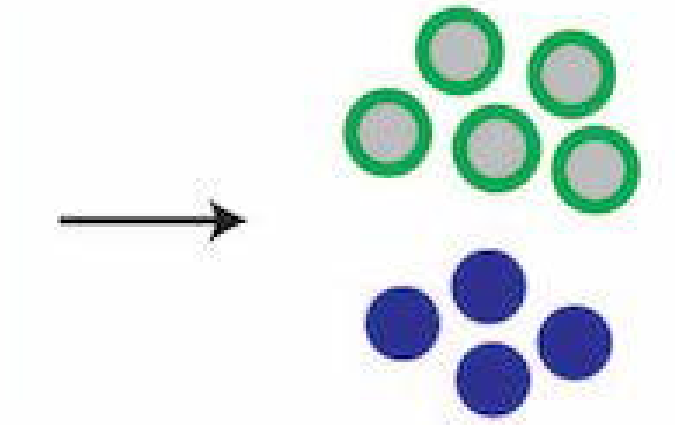

Nuclei sorting and molecular validation

\section{Population-derived SVMs, nonlinear}
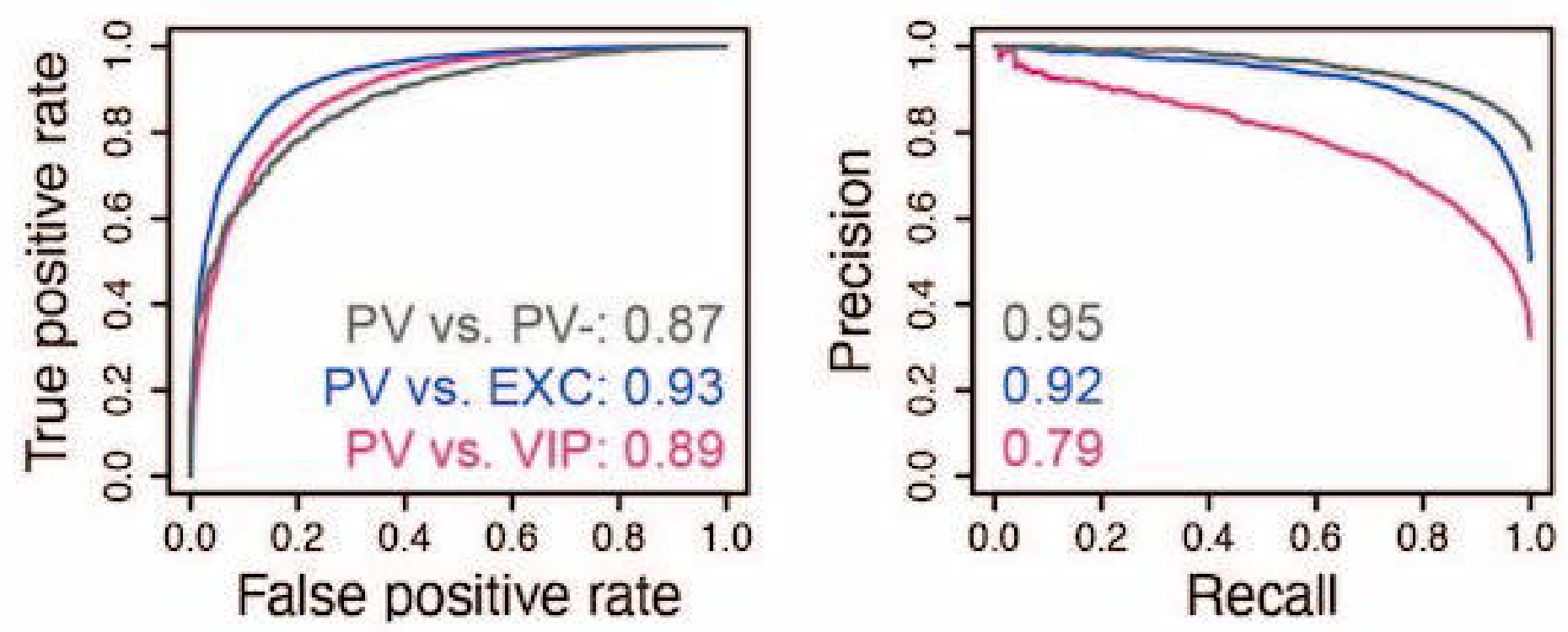

e

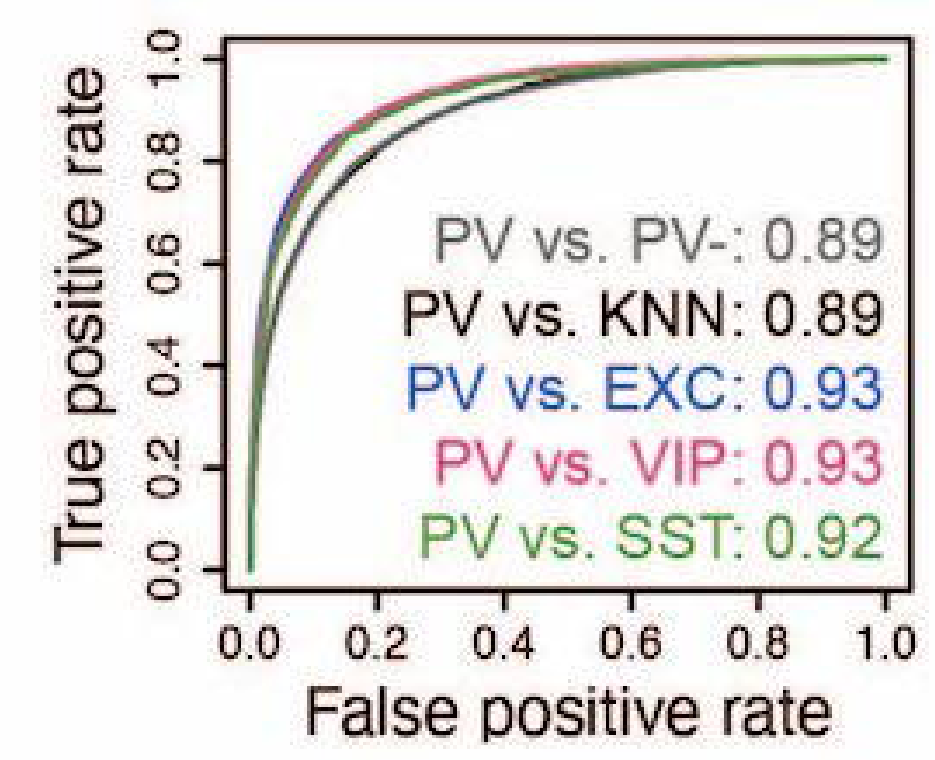

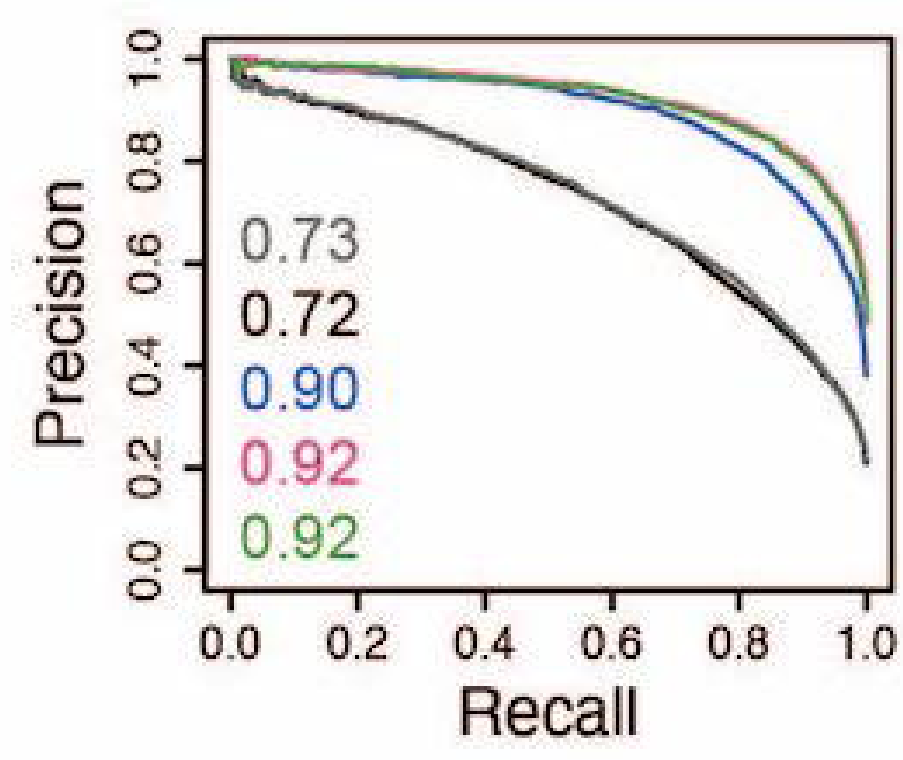

PV vs. EXC SVM TECCT Rora AATCA Lhx6 CACT: Tfe2 TCACA Maf IATTT,Mef2C
PV vs. VIP SVM AfsaA Elf5 CTT_. Nkx28 AATTA Nr2e3 EdT.Ac Stat6 T.ACCT $T_{\text {L }}$ Err3

( 


\section{a \\ bioRxiv preprint doi: https://doi.org/10.1101/2021.04.15.439984; this version posted April 15, 2021. The copyright holder for this preprint (which} was not certified by peer review) is the author/funder, who has granted bioRxiv a license to display fhe preprinf in perpefuity. It is made
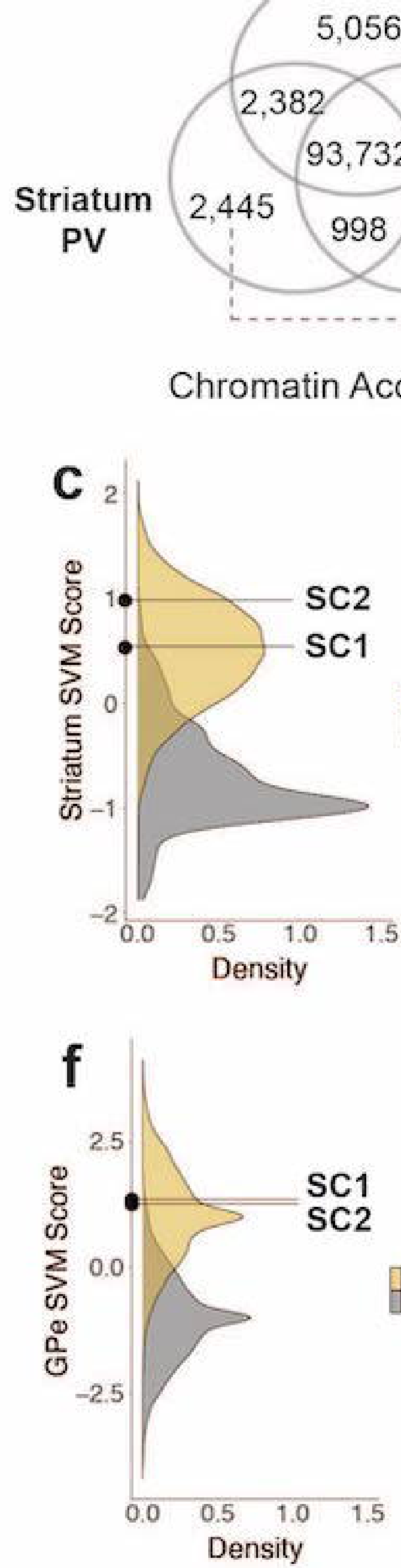

d Striatum PCA

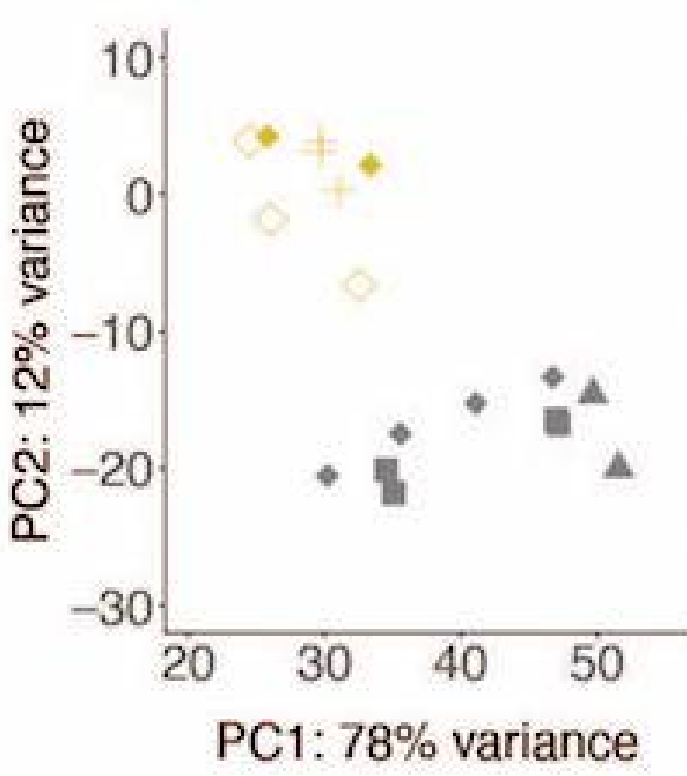

I PV

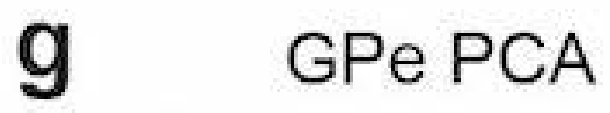

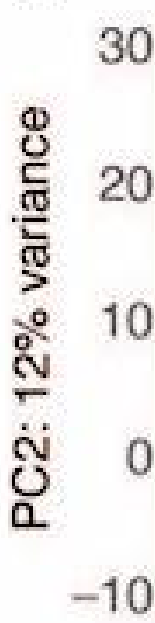

$\begin{array}{llll}10 & 20 & 30 & 40\end{array}$

PC1: $78 \%$ variance
Target Cell Type

PV-

Virus

e

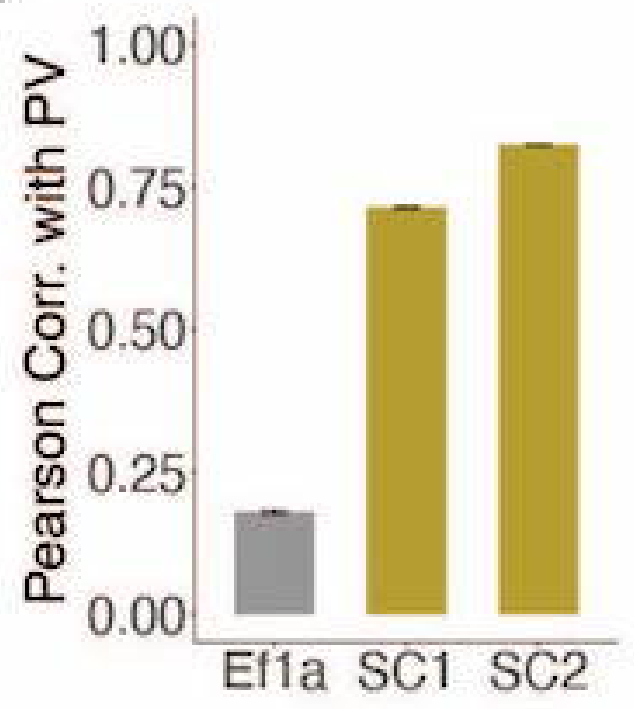

h

Target Cell Type

PV-

Virus

- cSNAAlL

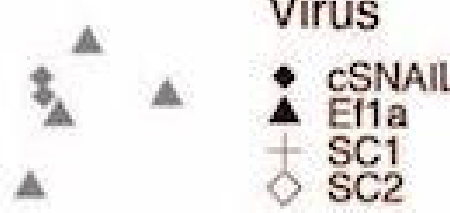

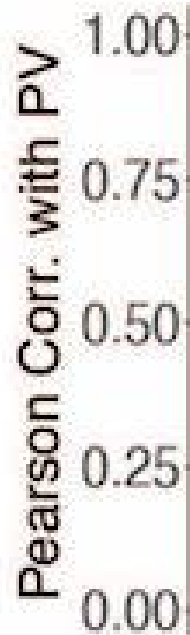

Ef1a SC1 SC2 


\section{Model}

- PV vs. PV- (pop)

- PV vs. PV- (pop, with rbf)

- PV vs. PV- (sn)

- PV vs. KNN (sn)

- PV vs. EXC (pop)

- PV vs. EXC (pop, with rbf)

- PV vs. EXC (sn)

- PV vs. VIP (pop)

- PV vs. VIP (pop, with rbf)

- PV vs. VIP (sn)

- PV vs. SST (sn)

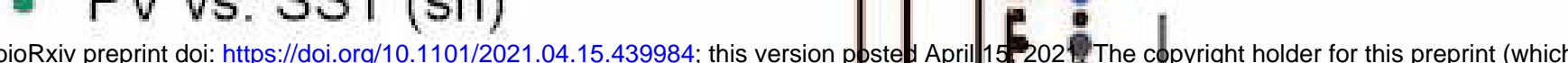

\section{Number of} supporting seqlets

- 1000

- 2000
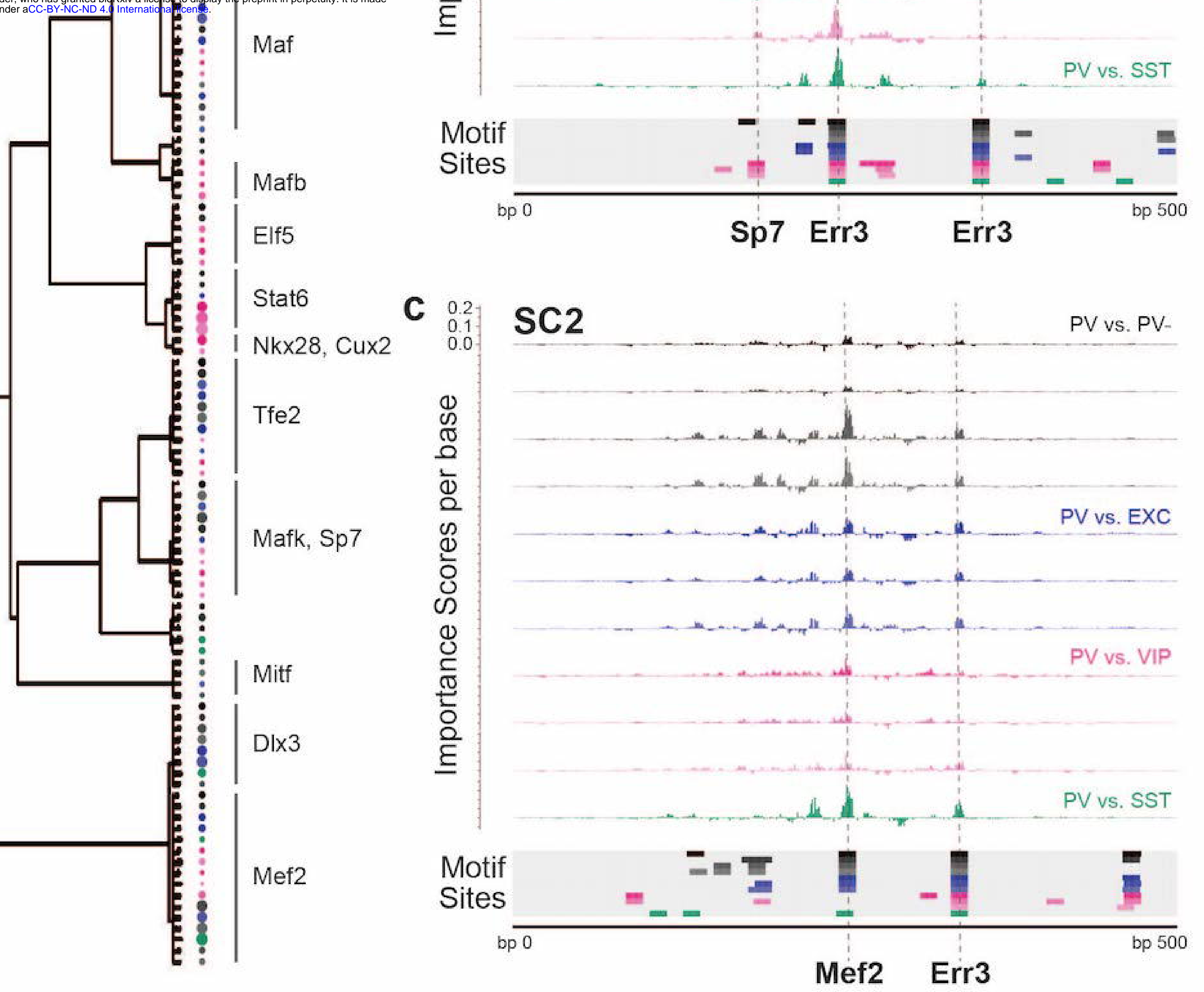